\title{
Semi-classical calculations of the two-point correlation form factor for diffractive systems
}

\author{
E. Bogomolny and O. Giraud \\ Laboratoire de Physique Théorique et Modèles Statistiques * \\ Université de Paris XI, Bât. 100 \\ 91405 Orsay Cedex, France
}

October 27, 2018

\begin{abstract}
The computation of the two-point correlation form factor $K(\tau)$ is performed for a rectangular billiard with a small size impurity inside for both periodic or Dirichlet boundary conditions. It is demonstrated that all terms of perturbation expansion of this form factor in powers of $\tau$ can be computed directly from a semiclassical trace formula. The main part of the calculation is the summation of non-diagonal terms in the cross product of classical orbits. When the diffraction coefficient for the scattering on the impurity is a constant our results coincide with the expansion of exact expressions obtained in Ref. [1] by a different method.
\end{abstract}

*Unité Mixte de Recherche de l'Université Paris XI et du CNRS (UMR 8626) 


\section{Introduction}

It is widely accepted that statistical properties of the energy levels of a quantum problem are determined mainly by the nature of its classical motion. Thus spectral statistics of classically chaotic systems follow the random matrix predictions [2], [3] and spectral statistics of classically integrable models agree with the Poisson statistics [4]. A lot of numerical evidence (see e.g. [5]) and partial analytical arguments [6]-9] strongly support these conjectures.

But there are models which (for various reasons) do not fit into the above scheme. A noticeable example is given by the so-called diffractive systems where one or a few small-size scatters are added to an otherwise smooth model. In classical mechanics point-like singularities affect only a zero-measure set of trajectories which directly hit them but in quantum mechanics singularities often lead to important effects. Though the addition of a scatter to a chaotic system has no effect on its spectral statistics 10 even one small-size impurity changes completely the statistical properties of integrable models [11].

The difference between chaotic and integrable models can intuitively be understood as follows. In chaotic systems classical trajectories and quantum wave functions cover the whole surface of constant energy and the addition of a few centers of scattering can not change this property. On the other hand, in integrable models trajectories and wave functions are confined to invariant tori and small-size scatters will, in general, induce transitions between different tori, qualitatively changing the nature of motion.

The investigation of integrable models with short-range scatters has been initiated in Ref. [11] (see also [12 and references therein) and recently attracts wide attention from both experimental and theoretical points of view. Experimentally, non-hydrogenic atoms in external fields [13] are just a possible realization of such models. Theoretically, these models are rare examples where spectral statistics can be computed analytically.

In Ref. [1] the two-point correlation function for a rectangular billiard with a delta-function potential has been computed exactly and in 14 the nearestneighbor distributions for this model were obtained. The main conclusion of these works is that the spectral statistics of diffractive systems belong to the so-called intermediate type. Intermediate spectral statistics describes level repulsion like random matrix ensembles but the nearest-neighbor distribution has exponential decrease like Poisson statistics. These mixed properties 
of diffractive systems are similar to spectral statistics of the 3-dimensional Anderson model at the metal-insulator transition point [15].

The purpose of this paper is the investigation of spectral statistics of diffractive systems by semiclassical methods. Our starting point is the trace formula for diffractive systems derived in [16] and [17] which allows to express the Green function and all related quantities as a sum over periodic and diffractive orbits. The two-point correlation function is obtained as usual by smoothing the product of 2 densities of states over a small energy interval. It has been shown in [18] that the linear on $\tau$ term in the expansion of the two-point correlation form factor $K(\tau)$ can be obtained by a diagonal approximation, which consists of taking into account only contributions from pairs of orbits with exactly the same length, and summing them using the Hannay-Ozorio de Almeida sum rule [19].

The main difficulty of obtaining the higher order terms of the expansion of $K(\tau)$ in powers of $\tau$ is the necessity to take into account non-diagonal contributions of classical orbits with slightly different actions. The method which permits to solve this problem for integrable systems has been proposed in [20]. Using and simplifying it we construct all terms of perturbation series expansion of the two-point correlation form factor $K(\tau)$ into series of $\tau$ for rectangular billiard with a delta-function impurity for both periodic and Dirichlet boundary conditions. The results agree with the expansion of exact formulas obtained in [1].

The model with a delta-function potential is characterized by a constant diffraction coefficient. It is of interest to consider more general models with a non-constant diffraction coefficient. Though the exact solution of such models are not known we construct the perturbation series expansion of $K(\tau)$ in this case as well.

The plan of the paper is the following. The trace formula for diffractive systems is shortly discussed in Section 2. In Section 3 the detailed discussion of perturbation series for the form factor of a rectangular billiard with delta-function potential and Dirichlet boundary conditions is presented. The main part of this section is devoted to the summation of non-diagonal contributions. In Section 1 the case of Dirichlet boundary conditions is discussed and in Section 5 the case of arbitrary diffraction coefficient is considered. The expansion of the exact expression of $K(\tau)$ for rectangular billiards with constant diffraction coefficient obtained in [1] is derived in Appendix. 


\section{Trace formula for diffractive systems}

The starting point of the modern semiclassical approximation for multi-dimensional quantum systems is the semiclassical approximation for the (advanced) Green function as a sum over classical trajectories with energy $E$ starting from initial point $\vec{x}$ with momentum in the direction $\vec{n}$ and ending at final point $\vec{y}$ with the momentum in the direction $\vec{n}^{\prime}$ [21, 22,

$$
G(\vec{x}, \vec{y})=\sum_{t r} G\left((\vec{x}, \vec{n}),\left(\vec{y}, \vec{n}^{\prime}\right)\right)
$$

where the contribution from each trajectory has the form

$$
G\left((\vec{x}, \vec{n}),\left(\vec{y}, \vec{n}^{\prime}\right)\right)=A_{t r} \exp \left(\frac{i}{\hbar} S_{c l}-i \frac{\pi}{2} \nu\right) .
$$

$S_{c l}=S_{c l}(E, \vec{x}, \vec{y})$ is the classical action computed along the trajectory,

$$
A_{t r}=\frac{m}{i \hbar(2 \pi \hbar)^{\frac{1}{2}(f-1)}}\left|\frac{1}{k k^{\prime}} \operatorname{det} \frac{\partial^{2} S_{c l}}{\partial y \partial y^{\prime}}\right|^{\frac{1}{2}}
$$

where $m$ is the mass, $f$ is the dimension of the space, $y$ and $y^{\prime}$ are coordinates perpendicular to the trajectory (respectively at initial and final point), $k$ and $k^{\prime}$ are initial and final momenta, and $\nu$ is a phase (the Maslov index) which, roughly speaking, counts points where simple semiclassical approximation breaks down. Of course, variables $(\vec{x}, \vec{n})$ and $\left(\vec{y}, \vec{n}^{\prime}\right)$ are not all independent, and the sum (1) over classical trajectories must take into account, for each given starting point and momentum $(\vec{x}, \vec{n})$, all classically allowed final points and momenta.

For 2-dimensional free motion these formulas take especially simple form and the free Green function (in the units $m=\frac{1}{2}$ and $\hbar=1$ ) reads (see e.g. [24])

$$
G(\vec{x}, \vec{y})=\sum_{t r} \frac{e^{i k l-i \frac{\pi}{2} \nu-i \frac{3 \pi}{4}}}{\sqrt{8 \pi k l}}
$$

where $l$ is the length of the trajectory. 
The knowledge of the Green function permits to compute other quantum quantities. In particular the quantum density of states

$$
d(E)=\sum_{n} \delta\left(E-E_{n}\right)
$$

may formally be written by the means of the advanced Green function as

$$
d(E)=-\frac{1}{\pi} \mathcal{I} m \int d \vec{x} G(\vec{x}, \vec{x})
$$

The contribution from very short trajectories gives the mean level density, $\bar{d}$, and the integration of (4) over the whole space selects periodic orbit contributions [21]-23] and determines an oscillating part of level density

$$
d(E)=\bar{d}+d^{(o s c)}(E)
$$

For any 2-dimensional billiard

$$
\bar{d}=\frac{\mathcal{A}}{4 \pi}
$$

where $\mathcal{A}$ is the area of the billiard. The explicit form of $d^{(o s c)}(E)$ depends of the system considered (see e.g. [21]-23]).

Diffractive systems discussed in the paper are characterized by the existence of singularities which make the classical motion undetermined. Each time a classical trajectory hits a singularity there is no unique way to continue it. Quantum mechanics smoothes out these singularities and associates with each (not too strong) singularity a diffraction coefficient, $\mathcal{D}\left(\vec{n}, \vec{n}^{\prime}\right)$, which determines a scattering amplitude on this singularity from the initial direction $\vec{n}$ to the final direction $\vec{n}^{\prime}$. In the presence of a singularity the Green function in the whole space has two contributions

$$
G\left(\vec{x}, \vec{x}^{\prime}\right)=G_{0}\left(\vec{x}, \vec{x}^{\prime}\right)+G_{d}\left(\vec{x}, \vec{x}^{\prime}\right)
$$

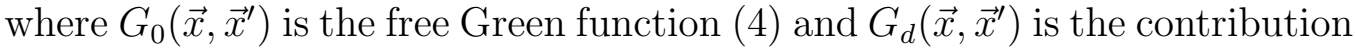
of trajectories that hit the singularity

$$
G_{d}\left(\vec{x}, \vec{x}^{\prime}\right)=\sum_{\vec{n}, \vec{n}^{\prime}} G_{0}\left(\vec{x},\left(\vec{x}_{0}, \vec{n}\right)\right) \mathcal{D}\left(\vec{n}, \vec{n}^{\prime}\right) G_{0}\left(\left(\vec{x}_{0}, \vec{n}^{\prime}\right), \vec{x}^{\prime}\right)
$$


where $\mathcal{D}\left(\vec{n}, \vec{n}^{\prime}\right)$ defined by Eq. (10) is called the diffraction coefficient. Here the quantity $G_{0}\left(\vec{x},\left(\vec{x}_{0}, \vec{n}\right)\right)$ is a contribution to the Green function from a classical trajectory starting at point $\vec{x}$ and ending at the singularity $\vec{x}_{0}$ with momentum in the direction $\vec{n} ; G_{0}\left(\left(\vec{x}_{0}, \vec{n}^{\prime}\right), \vec{x}^{\prime}\right)$ is a contribution to the Green function from a classical trajectory starting at point $\vec{x}_{0}$ with momentum in the direction $\vec{n}^{\prime}$ and ending at point $\vec{x}^{\prime}$.

For diffractive systems the density of states (6) can be written as the sum of three terms [17, 25, [16]

$$
d(E)=\bar{d}+d_{\text {p.o. }}(E)+d_{\text {d.o. }}(E),
$$

where $\bar{d}$ is the mean level density, $d_{\text {p.o. }}$ is the contribution of periodic orbits without singularity, and the third term, $d_{\text {d.o. }}(E)$, is a contribution from all classical trajectories starting and ending at the singularity, called diffractive orbits

$$
d_{d . o .}(E)=\sum_{m=1}^{\infty} \frac{1}{\pi m} \frac{\partial}{\partial E} \mathcal{I} m \sum_{\vec{n}_{i}, \vec{n}_{j}^{\prime}} G\left(\vec{n}_{1}, \vec{n}_{1}^{\prime}\right) \mathcal{D}\left(\vec{n}_{1}^{\prime}, \vec{n}_{2}\right) \ldots G\left(\vec{n}_{m}, \vec{n}_{m}^{\prime}\right) \mathcal{D}\left(\vec{n}_{m}^{\prime}, \vec{n}_{1}\right)
$$

where $G\left(\vec{n}, \vec{n}^{\prime}\right)$ is now the contribution to the Green function from a classical trajectory starting at the singular point with initial momentum in direction $\vec{n}$ and ending at it with final momentum in direction $\vec{n}^{\prime}$.

For elastic scattering the diffraction coefficient cannot be arbitrary but has to obey the optical theorem which is the manifestation of the quantum mechanical unitarity. In the most general form the optical theorem is 26]

$$
\mathcal{D}\left(\vec{n}, \vec{n}^{\prime}\right)-\overline{\mathcal{D}}\left(\vec{n}^{\prime}, \vec{n}\right)=-\frac{i}{4 \pi} \int \mathcal{D}\left(\vec{n}, \vec{n}^{\prime \prime}\right) \overline{\mathcal{D}}\left(\vec{n}^{\prime}, \vec{n}^{\prime \prime}\right) d o^{\prime \prime}
$$

where $d o^{\prime \prime}$ is the angle giving the direction of $\vec{n}^{\prime \prime}$.

In particular, a constant diffraction coefficient should have the form

$$
\mathcal{D}=\frac{\lambda}{1+\frac{i}{4} \lambda}
$$

with real $\lambda$. 


\section{Periodic boundary conditions}

Let us consider a rectangular billiard with sides $a$ and $b$ and with a pointlike scatter at a point $\left(x_{0}, y_{0}\right)$ inside the rectangle. We always assume that the ratio $a^{2} / b^{2}$ is a 'good' (diophantine) irrational number. This generic requirement is necessary to avoid 'accidental' degeneracies of periodic orbit lengths and under this condition it is possible to prove 9] that the two-point correlation function of such a rectangular billiard in the "free" case (that is without scatter) agrees with the Poisson statistics. In this section we impose periodic boundary conditions.

Fig. 1 shows a classical periodic orbit in the rectangle. Since opposite sides of the rectangle are identified, one can unfold a classical trajectory to a straight line on the plane tiled with rectangles; the images of the point-like scatter have coordinates $\left\{x_{0}+M a, y_{0}+N b\right\}, M, N \in \mathbb{Z}$.

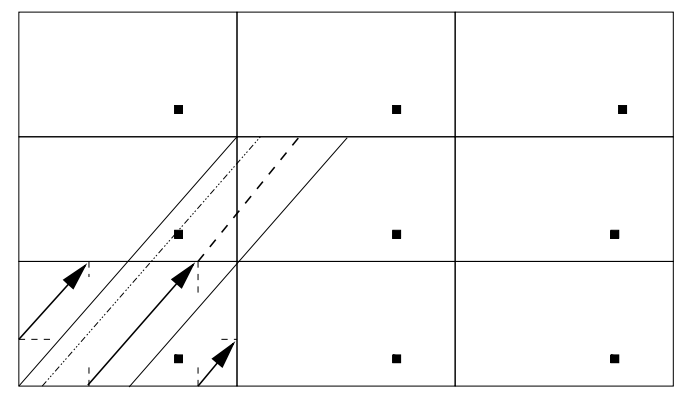

- Scattering point

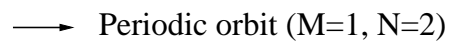

- - - The same orbit unfolded

-........ Diffractive orbit

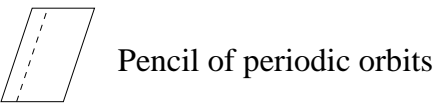

Figure 1: An unfolded trajectory in the rectangle

The unperturbed energy levels of a rectangular billiard with periodic boundary conditions are

$$
E=\left(\frac{2 \pi}{a} n\right)^{2}+\left(\frac{2 \pi}{b} m\right)^{2}
$$

for all integers $m, n=0, \pm 1, \pm 2, \ldots$.

When $m n \neq 0$ energy levels have multiplicity 4 . To remove this trivial degeneracy it is convenient to consider non-degenerate states only. Asymptotically it gives the factor $1 / 4$ in the periodic orbit density of states and 
to be consistent with Ref. [20] and Equations (6) and (10) it is necessary to multiply the diffraction coefficient by $g=4$ (see below).

Therefore in our case the smooth part will be

$$
\bar{d}=\frac{\mathcal{A}}{16 \pi}
$$

where $\mathcal{A}=a b$ is the area of the rectangle. The contribution of the periodic orbits to the density of states is given by [23]

$$
d_{\text {p.o. }}(E)=\sum_{p^{+}} \frac{\mathcal{A}_{p}}{2 \pi} \frac{1}{\sqrt{8 \pi k l_{p}}} e^{i k l_{p}-i \frac{\pi}{2} \nu_{p}-i \frac{\pi}{4}}+\text { c.c. }
$$

where

$$
l_{p}=\sqrt{(M a)^{2}+(N b)^{2}}
$$

is the length of a periodic orbit. In all integrable billiards periodic orbits are not isolated but belong to families which cover an area $\mathcal{A}_{p}$ (see Fig. 1). For the rectangular billiard with periodic boundary conditions the area covered by any family of periodic orbits is $\mathcal{A}_{p}=\mathcal{A}$. Since $d_{\text {p.o. }}(E)$ corresponds to the non-degenerate density of states, the summation in (17) is performed over all periodic orbits of length $l_{p}$ with $M, N \geq 0$ (which corresponds to divide by 4 the density (6)); this is expressed by the index $p^{+}$.

\subsection{Diffractive density of states}

The third term in the density (111) is the "density of diffractive orbits" (12), that is the contribution of classical trajectories starting and ending at the singularity. The diffraction coefficient for our model is a constant given by (14). The Green functions in (12) can be expressed as a sum over all diffractive orbits, that is over all vectors

$$
\overrightarrow{l_{d}}=(M a, N b)
$$

linking two images of the scatter in the plane tiled with rectangles:

$$
G=\sum_{\vec{n}, \vec{n}^{\prime}} G\left(\vec{n}, \vec{n}^{\prime}\right)=\sum_{\vec{l}_{d}} \frac{e^{i k l_{d}-i \frac{\pi}{2} \nu_{d}-i \frac{3 \pi}{4}}}{\sqrt{8 \pi k l_{d}}},
$$


where $l_{d}$ is given by expression identical to (18)

$$
l_{d}=\sqrt{(M a)^{2}+(N b)^{2}} .
$$

Note that when $M N \neq 0$ there are $g=4$ diffractive orbits with exactly the same lengths corresponding to $\pm M, \pm N$, As the Green functions enter in the diffractive trace formula (12) always multiplied by the diffraction coefficient it is convenient to restrict the summation in (20) to $M, N \geq 0$ and to multiply the diffraction coefficient by $g=4$.

Each term in (12) involving $m$ Green functions gives the following contribution to the density of states

$$
\begin{aligned}
d_{\text {d.o. }}^{(m)}(E) & =\frac{(g \mathcal{D})^{m}}{4 i m \pi k} \frac{\partial}{\partial k}\left(\sum_{\vec{n}, \vec{n}^{\prime}} G\left(\vec{n}, \vec{n}^{\prime}\right)\right)^{m}+\text { c.c. } \\
& =\frac{(g \mathcal{D})^{m}}{4 i m \pi k} \frac{\partial}{\partial k} \sum_{\vec{l}_{1}, \ldots, \vec{l}_{m}} \frac{e^{i k l_{1}-3 i \pi / 4}}{\sqrt{8 \pi k l_{1}}} \ldots \frac{e^{i k l_{m}-3 i \pi / 4}}{\sqrt{8 \pi k l_{m}}}+\text { c.c. }
\end{aligned}
$$

$\vec{l}_{1}, \ldots, \vec{l}_{m}$ are $m$ diffractive orbits vectors and $l_{j}$ are their lengths. The Maslov index in the periodic case is equal to zero.

The method which permits to treat such sums has been proposed in [20]. Its main content is the existence in the sums over orbits $\vec{l}_{j}$ of saddle-point manifolds which correspond to vectors $\vec{l}_{j}$ almost parallel to a given vector $\vec{L}$ (see Fig. 2).

When the $m^{\text {th }}$ power of the Green function, $G^{m}$, is considered we can distinguish different saddle-point manifolds [20]. The first corresponds to the case when all $m$ vectors $\vec{l}_{1}, \ldots, \vec{l}_{m}$ are close to a unique fixed vector $\vec{L}$. The others are given by contributions where the $\vec{l}_{j}, 1 \leq j \leq m$, are gathered into $p$ groups $(2 \leq p \leq m)$ with the following properties:

(i) within each group all vectors are almost parallel to a vector $\vec{L}_{i}$

(ii) the $p$ vectors $\vec{L}_{i}$ are quite far from each other (and in particular their lengths are different).

Let us first order vectors $\vec{L}_{1}, \ldots, \vec{L}_{p}$ by a certain manner, e.g. according to their lengths

$$
L_{1}<L_{2}<\ldots<L_{p} .
$$




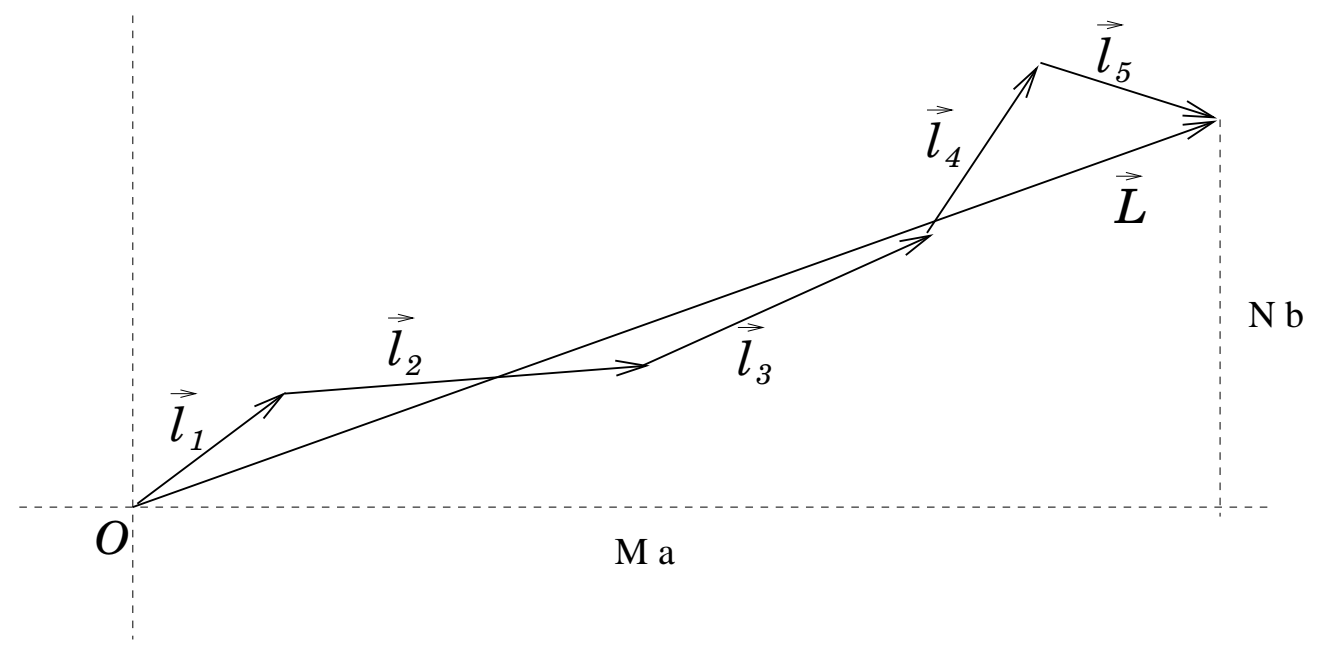

Figure 2: A saddle-point contribution to $G^{m}(\vec{L})$.

Because it is assumed that all $L_{j}$ are different it is always possible.

Then following [20] one can write the formal expression

$$
G^{m}=\sum_{p=1}^{m} \sum_{\vec{L}_{1}, \ldots, \vec{L}_{p}} G_{p}^{m}\left(\vec{L}_{1}, \ldots, \vec{L}_{p}\right),
$$

where $G$ is given by $(20)$ and

$$
\begin{aligned}
& G_{p}^{m}\left(\vec{L}_{1}, \ldots, \vec{L}_{p}\right)= \\
& \quad \sum_{\substack{m_{1}+\ldots+m_{p}=m \\
m_{i} \geq 1}} N\left(m_{1}, \ldots, m_{p}\right) \sum_{\vec{l}_{1}, \ldots, \vec{l}_{m}} \frac{e^{i k l_{1}-3 i \pi / 4}}{\sqrt{8 \pi k l_{1}}} \ldots \frac{e^{i k l_{m}-3 i \pi / 4}}{\sqrt{8 \pi k l_{m}}} \\
& \times \delta\left(\vec{l}_{1}+\ldots+\vec{l}_{m_{1}}-\vec{L}_{1}\right) \ldots \delta\left(\vec{l}_{m_{1}+\ldots+m_{p-1}+1}+\ldots+\vec{l}_{m}-\vec{L}_{p}\right)
\end{aligned}
$$

for any partition $m_{1}+\ldots+m_{p}=m$ of $m$ into a sum of $p$ integers It is assumed that vectors $\vec{l}_{j}$ all have positive components. $N\left(m_{1}, \ldots, m_{p}\right)$ is the number of possible permutations of the $l_{j}$ and will be discussed later.

The meaning of this representation is the following (for more details see [20]). The amplitudes corresponding to vectors almost parallel (i.e. belonging 
to the same group) should be summed coherently but the ones with different vectors $\vec{L}_{j}$ (that is from different groups) are non-coherent. Therefore after a smoothing over a small energy window only the square of the amplitudes of non-coherent contributions will survive.

Let us consider at first the case where $m=2$ and $p=1$ (see Fig. 3)

$$
G_{1}^{2}(\vec{L})=\sum_{\vec{l}_{1}, \vec{l}_{2}} \frac{e^{i k l_{1}-3 i \pi / 4}}{\sqrt{8 \pi k l_{1}}} \frac{e^{i k l_{2}-3 i \pi / 4}}{\sqrt{8 \pi k l_{2}}} \delta\left(\overrightarrow{l_{1}}+\overrightarrow{l_{2}}-\vec{L}\right)
$$

(in this case the symmetry factor $N(2)$ is equal to 1 ). We have to sum

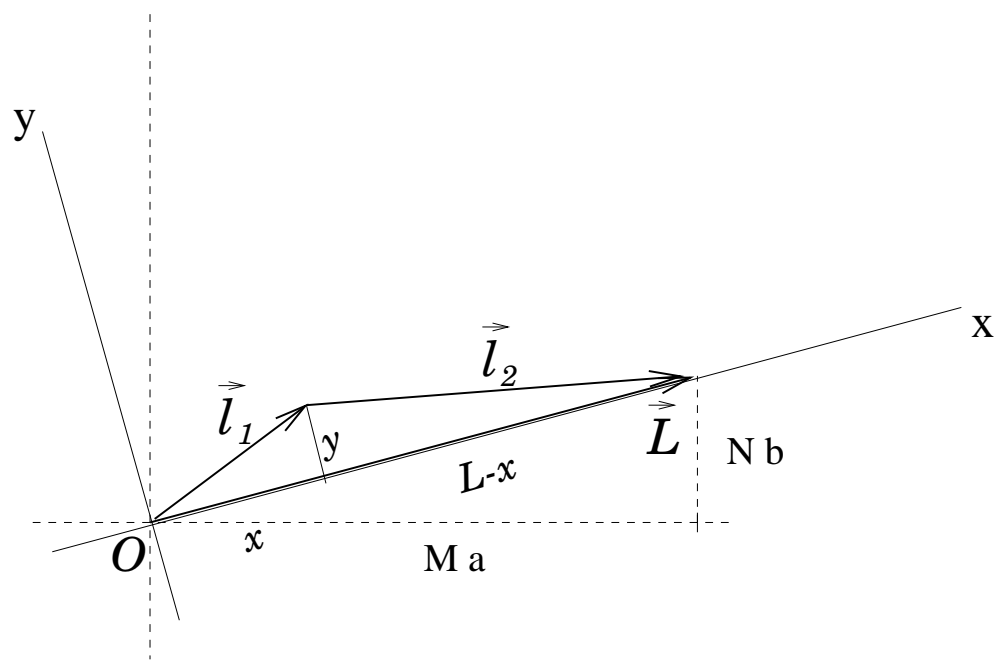

Figure 3: Contributions to the product of two Green functions

products of 2 Green functions over all vectors $\vec{l}_{1}=\left(m_{1} a, n_{1} b\right)$ and $\vec{l}_{2}=$ $\left(m_{2} a, n_{2} b\right)$ verifying the condition

$$
\overrightarrow{l_{1}}+\overrightarrow{l_{2}}=\vec{L}
$$

It means that we sum over all positive integers $m_{1}, n_{1}, m_{2}, n_{2}$ such that this condition is verified. Changing the sum into an integral, we have to integrate over $m_{i}$ and $n_{i}(i=1,2)$. In the coordinates $(x, y)$ where $\vec{L}=(0, L)$ (see Fig. 
3) we set $\overrightarrow{l_{1}}=(x, y)$, so that $\vec{l}_{2}=(L-x, y)$ : since $d m_{1} d n_{1}=d x d y / \mathcal{A}$, we get

$$
\begin{aligned}
G_{1}^{2}(\vec{L}) & =\int d m_{i} d n_{i} \frac{e^{i k l_{1}-3 i \pi / 4}}{\sqrt{8 \pi k l_{1}}} \frac{e^{i k l_{2}-3 i \pi / 4}}{\sqrt{8 \pi k l_{2}}} \delta\left(\vec{l}_{1}+\vec{l}_{2}-\vec{L}\right) \\
& =\frac{1}{\mathcal{A}} \int_{0}^{L} d x \int_{-\infty}^{\infty} d y \frac{e^{i k l_{1}-3 i \pi / 4}}{\sqrt{8 \pi k l_{1}}} \frac{e^{i k l_{2}-3 i \pi / 4}}{\sqrt{8 \pi k l_{2}}}
\end{aligned}
$$

(the condition $0 \leq x \leq L$ is necessary to have $\vec{l}_{1}$ and $\vec{l}_{2}$ in the upper right quadrant). In the semi-classical limit where $k \rightarrow \infty$ we use

$$
l_{1}+l_{2} \simeq L+\frac{L}{2 x(L-x)} y^{2}
$$

and after performing the integrals

$$
G_{1}^{2}(\vec{L})=\frac{L}{2 i k \mathcal{A}} \frac{e^{i k L-3 i \pi / 4}}{\sqrt{8 \pi k L}} .
$$

More generally this method can be iterated to get

$$
G_{1}^{m}(\vec{L})=\left(\frac{L}{2 i k \mathcal{A}}\right)^{m-1} \frac{1}{(m-1) !} \frac{e^{i k L-3 i \pi / 4}}{\sqrt{8 \pi k L}} .
$$

The saddle-point manifold in this case is the space of sets of $m$ vectors almost parallel to $\vec{L}$ such that their sum is equal to $\vec{L}$ (see Fig. 2). This result can also be obtained almost without calculations by using Stokes' theorem.

It may be noticed that the part of the Green function corresponding to the sum of contributions $G_{1}^{m}, \sum_{m=1}^{\infty}(g \mathcal{D})^{m-1} G_{1}^{m}(\vec{L})$, is the perturbation series expansion of the Green function and from (31) one gets

$$
\sum_{m=1}^{\infty}(g \mathcal{D})^{m-1} G_{1}^{m}(\vec{L})=\exp \left(\frac{\rho g \mathcal{D}}{2 i k} L\right) G(L)
$$

where $\rho=1 / \mathcal{A}$ is the density of scatters and

$$
G(L)=\frac{e^{i k L-3 i \pi / 4}}{\sqrt{8 \pi k L}}
$$


is the usual contribution to the free Green function of a classical trajectory of length $L$. The result (32) is exactly the exponential attenuation one expects for the coherent propagation of a particle in a diffractive medium with coefficient of diffraction $\mathcal{D}$.

The terms $G_{p}^{m}\left(\vec{L}_{1}, \ldots, \vec{L}_{p}\right)$ can be computed (see 20]) by the same method by considering all possible partitions of the $m$ vectors into $p$ groups of almost parallel vectors. But in this case a combinatorial factor which counts the exact degeneracies has to be taken into account. For a given partition of $m$ into a sum of $p$ integers $m_{1}, \ldots, m_{p}$

$$
m_{1}+\ldots+m_{p}=m
$$

we obtain a saddle-point manifold in the sum (25) by choosing $m_{1}$ vectors parallel to $\vec{L}_{1}, m_{2}$ vectors parallel to $\vec{L}_{2}$, and so on. But the numbers $m_{i}$ do not fix a diffractive trajectory uniquely. Trajectories built from the same set of primitive diffractive orbits connected in a different order have exactly the same lengths and numbers $m_{i}$.

All such trajectories correspond to one of the permutation of the following sequence of $p$ symbols

$$
\underbrace{1 \ldots 1}_{m_{1}} \underbrace{2 \ldots 2}_{m_{2}} \cdots \underbrace{p \ldots p}_{m_{p}}
$$

where it is assumed that in the sequence there exist $m_{1}$ symbols of type 1 , $m_{2}$ symbols of type 2 , etc, and finally $m_{p}$ symbols of type $p$. Each symbol $j$ corresponds to a different vector $\vec{L}_{j}$ defining a certain primitive diffractive orbit and a permutation of the sequence (35) describes how a composite diffractive orbit is built.

For example, at Fig. 1 trajectories with $m=4$ and $p=2$ are de-pictured. Fig. Ala corresponds to all partitions with $m_{1}=3$ and $m_{2}=1$

$$
1112112112112111
$$

and Fig. Tb represents partitions with $m_{1}=m_{2}=2$

$$
112212121221221121212112 .
$$

At these figures trajectories of types 1 and 2 are represented respectively by horizontal and vertical lines. Since the diffraction coefficient is a constant 


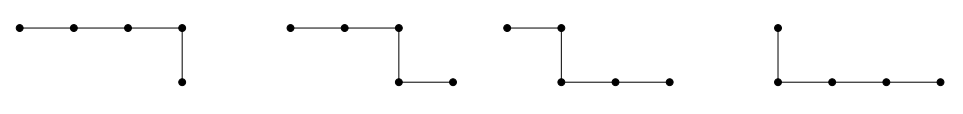

a)

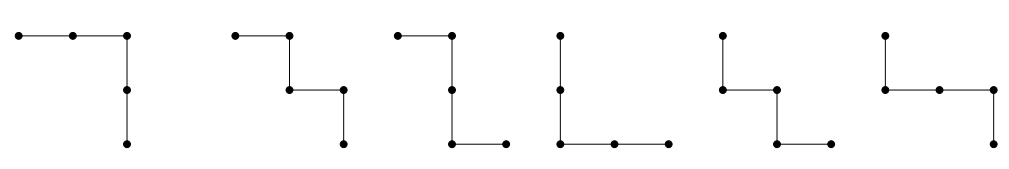

b)

Figure 4: Trajectories with $m=4$ and $p=2$ with exactly the same length. a) $m_{1}=3, m_{2}=1$; b) $m_{1}=2, m_{2}=2$.

the corresponding saddle-point manifolds (consisting of trajectories close to these exactly degenerate ones) all give the same contribution to the density $d_{\text {d.o. }}$; therefore the contribution from one saddle point has to be multiplied by their number which is the number of partitions of $m$ into $p$ groups with $m_{i}$ elements in the $i^{\text {th }}$ group

$$
N\left(m_{1}, \ldots, m_{p}\right)=\frac{\left(m_{1}+\ldots+m_{p}\right) !}{m_{1} ! \ldots m_{p} !} .
$$

These exact multiplicities of diffraction orbits clearly demonstrate the difference between scattering on regular and random arrays. For a random distribution of scattering points all trajectories as at Fig. 1 will have different lengths but, because in our model all points of diffraction are copies of the same scattering point, these trajectories have equal lengths. Finally

$$
G_{p}^{m}\left(\vec{L}_{1}, \ldots, \vec{L}_{p}\right)=\sum_{\substack{m_{1}+\ldots+m_{p}=m \\ m_{i} \geq 1}} \frac{m !}{m_{1} ! \ldots m_{p} !} \prod_{i=1}^{p}\left(\frac{L_{i}}{2 i k \mathcal{A}}\right)^{m_{i}-1} \frac{G\left(L_{i}\right)}{\left(m_{i}-1\right) !}
$$

where $G(L)$ is defined in (33).

According to Equation (22), the density of diffractive orbits is the derivative of the powers of the Green function. To get the leading term in the 
semiclassical approximation it is necessary to differentiate only the exponent and the diffractive density of states takes the form

$$
d_{\text {d.o. }}(E)=\sum_{p=1}^{\infty} \sum_{L_{1}<\ldots<L_{p}} d_{d . o .}^{(p)}\left(L_{1}, \ldots, L_{p}\right) G\left(L_{1}\right) \ldots G\left(L_{p}\right)+c . c .,
$$

where $\vec{L}_{1}, \ldots, \vec{L}_{p}$ are summed over the upper-right quadrant and

$$
\begin{aligned}
& d_{\text {d.o. }}^{(p)}\left(L_{1}, \ldots, L_{p}\right)=\frac{L_{1}+\ldots+L_{p}}{4 \pi k} \sum_{m=p}^{\infty} \frac{1}{m} \sum_{\substack{m_{1}+\ldots+m_{p}=m \\
m_{i} \geq 1}} R\left(m_{1}, \ldots, m_{p}\right) \\
& \times\left(\frac{L_{1}}{2 i k \mathcal{A}}\right)^{m_{1}-1} \frac{1}{\left(m_{1}-1\right) !} \ldots\left(\frac{L_{p}}{2 i k \mathcal{A}}\right)^{m_{p}-1} \frac{1}{\left(m_{p}-1\right) !}
\end{aligned}
$$

where

$$
R\left(m_{1}, \ldots, m_{p}\right)=(g \mathcal{D})^{m_{1}+\cdots+m_{p}} \frac{\left(m_{1}+\ldots+m_{p}\right) !}{m_{1} ! \ldots m_{p} !}
$$

$d_{d . o .}^{(p)}\left(L_{1}, \ldots, L_{p}\right)$ is the contribution of all diffractive trajectories (like those at Fig. 1 ) built from $m_{1}$ vectors $\vec{L}_{1}, m_{2}$ vectors $\vec{L}_{2}$, etc.

The term with $p=1$ is

$$
d_{\text {d.o. }}^{(1)}(L)=\frac{i \mathcal{A}}{2 \pi} \sum_{m=1}^{\infty} \frac{1}{m !}\left(\frac{g \mathcal{D} L}{2 i k \mathcal{A}}\right)^{m}=\frac{i \mathcal{A}}{2 \pi}\left(\exp \left(\frac{g \mathcal{D}}{2 i k \mathcal{A}} L\right)-1\right) .
$$

Since the contribution of periodic orbits (17) can be rewritten

$$
\sum_{L} \frac{i \mathcal{A}}{2 \pi} G(L)
$$

one gets, adding all contributions,

$$
d^{(o s c)}(E)=\sum_{p=1}^{\infty} \sum_{L_{1}<\ldots<L_{p}} d_{p}\left(L_{1}, \ldots, L_{p}\right) G\left(L_{1}\right) \ldots G\left(L_{p}\right)+c . c .
$$

where

$$
d_{1}(L)=\frac{i \mathcal{A}}{2 \pi} \exp \left(\frac{g \mathcal{D}}{2 i k \mathcal{A}} L\right),
$$

and $d_{p}\left(L_{1}, \ldots, L_{p}\right)=d_{d . o .}^{(p)}\left(L_{1}, \ldots, L_{p}\right)$ for $p \geq 2$. 


\subsection{Two-point correlation form factor}

The 2-point correlation function is related with the level density by the usual formula

$$
R_{2}(\epsilon)=\left\langle d\left(E+\frac{\epsilon}{2}\right) d\left(E-\frac{\epsilon}{2}\right)\right\rangle,
$$

where the brackets denote an energy averaging around $E$ over an interval of energy much larger than the mean level spacing $1 / \bar{d}$ and much smaller than energy $E$.

The two-point correlation form factor is defined as the Fourier transform of the connected part of $R_{2}(\epsilon)$ :

$$
K(\tau)=\int_{-\infty}^{\infty} \frac{d \epsilon}{\bar{d}}\left\langle d^{(o s c)}\left(E+\frac{\epsilon}{2}\right) d^{(o s c)}\left(E-\frac{\epsilon}{2}\right)\right\rangle e^{2 i \pi \bar{d} \epsilon \tau},
$$

(the factors are chosen so that $\tau$ and $K(\tau)$ are dimensionless).

The advantage of the representation (45) is that it is a sum over saddle-

points (each set of vectors $\left(\vec{L}_{1}, \ldots, \vec{L}_{p}\right)$ for $p \geq 1$ is a saddle-point) which are uncorrelated, so we can apply the usual diagonal approximation to this sum: in the cross product (48) only squares of terms corresponding to a given saddle-point will give a contribution.

Using the expansion of momentum $\sqrt{E+\epsilon} \simeq \sqrt{E}+\epsilon /(2 \sqrt{E})$ we get

$$
R_{2}(\epsilon)=\sum_{p=1}^{\infty} \frac{1}{(8 \pi k)^{p}} \sum_{L_{1}<\ldots<L_{p}}\left|d_{p}\left(L_{1}, \ldots, L_{p}\right)\right|^{2} \frac{e^{i \epsilon\left(L_{1}+\ldots+L_{p}\right) /(2 k)}}{L_{1} \cdots L_{p}}+\text { c.c. }
$$

where $d_{p}\left(L_{1}, \ldots, L_{p}\right)$ are defined in (45).

As all quantities depend only of the lengths of vectors $\vec{L}_{i}$ and $L_{i} \rightarrow \infty$ one can substitute the summation over $\vec{L}_{i}$ by the integration with the density

$$
\rho(l)=\int_{0}^{\infty} d M d N \delta\left(l-\sqrt{(M a)^{2}+(N b)^{2}}\right)=\frac{\pi l}{2 \mathcal{A}} .
$$

(Remind that we consider vectors in the upper-right quadrant with positive $M, N$.

Because

$$
\sum_{L_{1}<\ldots<L_{p}}=\frac{1}{p !} \sum_{L_{1}, \ldots, L_{p}}
$$


for any symmetric summand one obtains

$$
\begin{aligned}
R_{2}(\epsilon) & =\sum_{p=1}^{\infty} \frac{1}{p !} \int_{0}^{\infty} \frac{d L_{1}}{16 \mathcal{A} k} \ldots \int_{0}^{\infty} \frac{d L_{p}}{16 \mathcal{A} k}\left|d_{p}\left(L_{1}, \ldots, L_{p}\right)\right|^{2} e^{i \epsilon\left(L_{1}+\ldots+L_{p}\right) /(2 k)} \\
& + \text { c.c.. }
\end{aligned}
$$

The two-point correlation form factor has the similar form

$$
K(\tau)=\sum_{p=1}^{\infty} K_{p}(\tau)
$$

where

$$
\begin{aligned}
K_{p}(\tau) & =\frac{4 \pi k}{\bar{d} p !} \int_{0}^{\infty} \frac{d L_{1}}{16 \mathcal{A} k} \ldots \int_{0}^{\infty} \frac{d L_{p}}{16 \mathcal{A} k}\left|d_{p}\left(L_{1}, \ldots, L_{p}\right)\right|^{2} \\
& \times \delta\left(L_{1}+\ldots+L_{p}-4 \pi \bar{d} k \tau\right)
\end{aligned}
$$

and $\bar{d}=\mathcal{A} / 16 \pi$.

The term with $p=1$ is especially simple (see (46))

$$
K_{1}(\tau)=\left|\exp \left(-i \frac{\mathcal{D} g}{8} \tau\right)\right|^{2} .
$$

Using the optical theorem (13) this expression can be rewritten in the following form

$$
K_{1}(\tau)=\exp \left(-\frac{|\mathcal{D}|^{2} g}{16} \tau\right)
$$

Substituting (41) into (54) one obtains the contributions from terms with $p \geq 2$ (we define $\tau_{i}$ by $L_{i}=4 \pi k \bar{d} \tau_{i}$ )

$$
\begin{aligned}
& K_{p}(\tau)=\frac{\tau^{2}}{p !} \int_{0}^{\infty} d \tau_{1} \ldots \int_{0}^{\infty} d \tau_{p} \sum_{m, n \geq p} \sum_{\substack{m_{1}+\ldots+m_{p}=m \\
n_{1}+\ldots+n_{p}=n}} \frac{(8 i)^{-m}(-8 i)^{-n}}{m n} \\
& \times R\left(m_{1}, \ldots, m_{p}\right) \bar{R}\left(n_{1}, \ldots, n_{p}\right) \\
& \times\left[\prod_{i=1}^{p} \frac{\tau_{i}^{m_{i}+n_{i}-2}}{\left(m_{i}-1\right) !\left(n_{i}-1\right) !}\right] \delta\left(\tau_{1}+\ldots \tau_{p}-\tau\right)
\end{aligned}
$$


where all $m_{i}, n_{i} \geq 1$. The remaining integral has the form

$$
J(\tau)=\int_{0}^{\infty} d \tau_{1} \ldots d \tau_{p} \tau_{1}^{m_{1}+n_{1}-2} \ldots \tau_{p}^{m_{p}+n_{p}-2} \delta\left(\tau_{1}+\ldots+\tau_{p}-\tau\right)
$$

and can easily be computed by the Laplace transform

$$
J(\tau)=\frac{\tau^{m+n-p-1}}{(m+n-p-1) !} \prod_{r=1}^{p}\left(m_{r}+n_{r}-2\right) !
$$

With Eqs. (57) - (59) one obtains the contribution to the form factor

$$
\begin{aligned}
K_{p}(\tau) & =\frac{1}{p !} \sum_{m, n \geq p} \sum_{\substack{m_{1}+\ldots+m_{p}=m \\
m_{i} \geq 1}} \sum_{\substack{n_{1}+\ldots+n_{p}=n \\
n_{i} \geq 1}} \frac{1}{m n} R\left(m_{1}, \ldots, m_{p}\right) R\left(n_{1}, \ldots, n_{p}\right) \\
& \times \prod_{r=1}^{p}\left[\frac{\left(m_{r}+n_{r}-2\right) !}{\left(m_{r}-1\right) !\left(n_{r}-1\right) !}\right] \frac{(8 i)^{-m}(-8 i)^{-n}}{(m+n-p-1) !} \tau^{m+n-p+1} .
\end{aligned}
$$

Adding all factors together we finally get the complete perturbation series expansion of the two-point correlation form factor for rectangular billiard with a delta-function impurity and periodic boundary conditions

$$
\begin{aligned}
K(\tau) & =e^{-|\mathcal{D}|^{2} g \tau / 16} \\
& +\sum_{p=2}^{\infty} \frac{1}{p !} \sum_{m, n \geq 0} A_{m n p}\left(\frac{-i \mathcal{D} g}{8}\right)^{m+p}\left(\frac{i \overline{\mathcal{D}} g}{8}\right)^{n+p} \tau^{m+n+p+1}
\end{aligned}
$$

where we have defined the rational numbers

$$
A_{m n p}=\frac{(m+p-1) !(n+p-1) !}{(m+n+p-1) !} \sum_{m_{i}, n_{j} \geq 0} \prod_{i=1}^{p}\left[\frac{C_{m_{i}+n_{i}}^{m_{i}}}{\left(m_{i}+1\right) !\left(n_{i}+1\right) !}\right]
$$

(the sum is taken over all non-negative integers $m_{i}$ and $n_{j}$ verifying $m_{1}+$ $\cdots+m_{p}=m$ and $n_{1}+\cdots+n_{p}=n$ ). Remind that in our case $g=4$. This result coincides with the expansion of the exact formula derived in [1] (see Appendix).

When $\tau \rightarrow 0, K(\tau) \rightarrow 1$ as expected for generic integrable billiards 18 . 
The special case $\mathcal{D}=-4 i$ obtained for $\lambda \rightarrow \infty$ in (14) corresponds to spectral statistics of the star graphs [27] where Eq. (61) for this value of the diffraction coefficient yields

$$
K(\tau)=e^{-4 \tau}+\sum_{p=2}^{\infty} \frac{(-2)^{p-1}}{p !} \sum_{m, n \geq 0} A_{m n p}(-2 \tau)^{m+n+p+1}
$$

and can be derived by another method [28].

\section{Dirichlet boundary conditions}

Let us consider now the case where we impose Dirichlet conditions on boundary of a rectangular billiard which means that the eigenfunctions of the quantum problem vanish on the boundary. It is well known that for this boundary conditions the classical motion corresponds to the specular reflection on the boundary. For example, Fig. 5 shows a periodic orbit in the rectangle. One can unfold the trajectory to a straight line on the plane

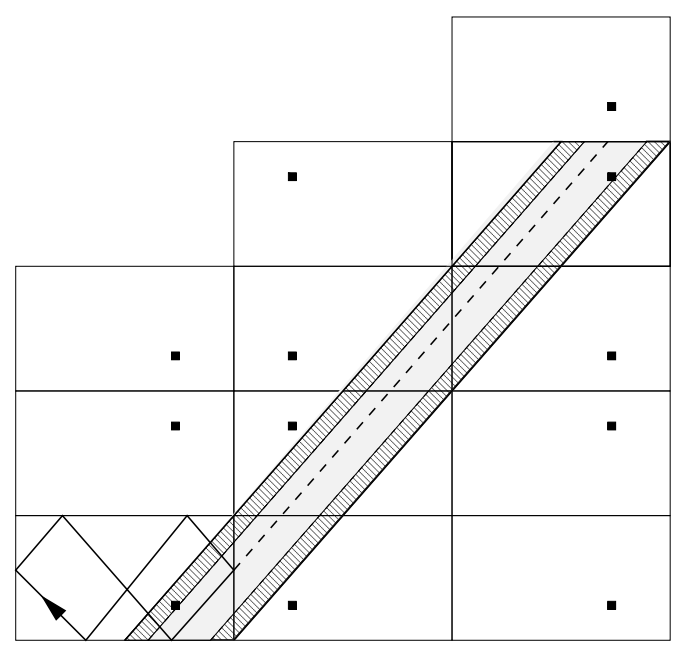

- Scatter

Periodic orbit $(\mathrm{M}=1, \mathrm{~N}=2)$

- - - The same orbit unfolded

$\mathbb{P}$ Pencil of periodic orbits

Figure 5: An unfolded trajectory in the rectangle

tiled with rectangles; the images of the point-like scatter have coordinates 
$\left\{\left(\epsilon_{1} x_{0}+2 M\right) a,\left(\epsilon_{2} y_{0}+2 N\right) b\right\}$, where as before $\left(x_{0}, y_{0}\right)$ are coordinates of the scatter, $M, N$ are in $\mathbb{Z}$ and $\epsilon_{1}$ and $\epsilon_{2}$ take the values \pm 1 .

The density of states still contains three terms

$$
d(E)=\bar{d}+d_{\text {p.o. }}(E)+d_{\text {d.o. }}(E)
$$

but in this case the sum (5) over the eigenvalues is not degenerate any more. Therefore the smooth part is

$$
\bar{d}=\frac{\mathcal{A}}{4 \pi}
$$

where $\mathcal{A}=a b$ is the area of the rectangle. The density of periodic orbits is given by an expression similar to (17):

$$
d_{\text {p.o. }}(E)=g \sum_{p^{+}} \frac{\mathcal{A}}{2 \pi} \frac{1}{\sqrt{8 \pi k l_{p}}} e^{i k l_{p}-i \frac{\pi}{2} \nu_{p}-i \frac{\pi}{4}}+\text { c.c. },
$$

where the summation is still performed over all periodic orbits of length $l_{p}$ with $M, N \geq 0$. But here

$$
l_{p}=\sqrt{(2 M a)^{2}+(2 N b)^{2}}
$$

and for the rectangular billiard with Dirichlet boundary conditions the multiplicity of periodic orbit lengths is $g=4$ for $M N \neq 0$.

The density of diffractive orbits is given by the usual formula (12) with diffraction coefficients (14). The Green function in (12) can be expressed as a sum over all diffractive orbits, that is all vectors linking two images of the scatter in the plane tiled with rectangles:

$$
G=\sum_{\vec{n}, \vec{n}^{\prime}} G\left(\vec{n}, \vec{n}^{\prime}\right)=\sum_{\vec{l}_{d}} \frac{e^{i k l_{d}-i \frac{\pi}{2} \nu_{d}-i \frac{3 \pi}{4}}}{\sqrt{8 \pi k l_{d}}}
$$

where the sum is taken over $M, N \in \mathbb{Z}$ since there is no degeneracy of the energy levels any more; $l_{d}$ is the length of a trajectory going from $\left(x_{0}, y_{0}\right)$ to an image of the scatter.

As in the periodic case we could restrict our investigation to $M, N \geq 0$ but now we have to consider the 16 vectors whose lengths are almost equal. Those are vectors linking $\left(x_{0}, y_{0}\right)$ to $\left( \pm 2 M a \pm x_{0}, \pm 2 N b \pm y_{0}\right)$. The lengths 
of these orbits are slightly different and can be expanded to the first order in $1 / M, 1 / N$. The vector linking $\left(x_{0}, y_{0}\right)$ to $\left(2 M a+\epsilon_{1} x_{0}, 2 N b+\epsilon_{2} y_{0}\right)$ (with $\left.\epsilon_{i}= \pm 1\right)$ has a length

$$
\begin{aligned}
l_{d} & =\sqrt{\left(2 M a+\left(\epsilon_{1}-1\right) x_{0}\right)^{2}+\left(2 N b+\left(\epsilon_{2}-1\right) y_{0}\right)^{2}} \\
& \simeq l_{p}+\left(\epsilon_{1}-1\right) \frac{2 M a x_{0}}{l_{p}}+\left(\epsilon_{2}-1\right) \frac{2 N b y_{0}}{l_{p}}
\end{aligned}
$$

where $l_{p}$ is given by (67). Taking into account that for this trajectory and the Dirichlet boundary conditions the phase $\exp \left(-\frac{1}{2} i \pi \nu_{d}\right)=\epsilon_{1} \epsilon_{2}$ the terms corresponding to a given $(M, N)$ in the Green function (68) can be gathered together. There are 16 type of such orbits corresponding to $\epsilon_{1}, \epsilon_{2}= \pm 1$ and all possible changes of signs of $M$ and $N$ (see Fig. 9). Their sum is

$$
\sum_{i=1}^{16} e^{i k l_{i}-i \frac{\pi}{2} \nu_{i}}=16 e^{i k l_{p}} \sin ^{2}\left(\phi_{1}\right) \sin ^{2}\left(\phi_{2}\right),
$$

where

$$
\begin{array}{r}
\phi_{1}=2 k \frac{M}{l_{p}} x_{0} a=k x_{0} \cos \varphi \\
\phi_{2}=2 k \frac{N}{l_{p}} y_{0} b=k y_{0} \sin \varphi
\end{array}
$$

and $\varphi$ is the angle between the periodic orbit vector $\vec{L}$ and the horizontal side of the rectangle. For Neumann boundary conditions sin in the above expression should be substituted by cos.

Therefore the difference between billiards with periodic boundary conditions considered in the previous Section and the Dirichlet case is the factor $r(\varphi)$ in $(68)$

$$
\sum_{\vec{n}, \vec{n}^{\prime}} G\left(\vec{n}, \vec{n}^{\prime}\right)=g \sum_{p} \frac{e^{i k l_{p}-i \frac{3 \pi}{4}}}{\sqrt{8 \pi k l_{p}}} r(\varphi),
$$

where the sum is taken over orbits with $M, N \geq 0$ and $g=4$. Here we have set

$$
r(\varphi)=4 \sin ^{2} \phi_{1}(\varphi) \sin ^{2} \phi_{2}(\varphi)
$$


Compared to the problem with periodic boundary conditions, the Green function (25) is modified by a product of factors $r\left(\varphi_{i}\right)$, where $\varphi_{i}$ is the angle between the periodic orbit $\vec{L}_{i}$ and the horizontal side of the rectangle. One can also leave the Green function unchanged but substitute the constant diffraction coefficient $\mathcal{D}$ by an effective coefficient, $S(\varphi)$, defined by

$$
S(\varphi)=\mathcal{D} r(\varphi)
$$

and taken at the saddle-point. The quantities $G_{p}^{m}\left(\vec{L}_{1}, \ldots, \vec{L}_{p}\right)$ defined by Eq. (25) are unchanged, and performing the same steps as in the previous Section one obtains the formula for the density of states quite similar to Eqs. (41), (45)

$$
d^{(o s c)}(E)=\sum_{p=1}^{\infty} d_{p}\left(L_{1}, \ldots, L_{p}\right) G\left(L_{1}\right) \ldots G\left(L_{p}\right)+\text { c.c. }
$$

where

$$
d_{1}(L)=\frac{i \mathcal{A}}{2 \pi} \exp (g \mathcal{D} r(\varphi) L /(2 i k \mathcal{A}))
$$

and $d_{p}\left(L_{1}, \ldots, L_{p}\right)$ is given by Eq. (41) with

$$
R\left(m_{1}, \ldots, m_{p}\right)=(g \mathcal{D})^{m_{1}+\cdots+m_{p}} \frac{\left(m_{1}+\ldots+m_{p}\right) !}{m_{1} ! \ldots m_{p} !} r\left(\varphi_{1}\right)^{m_{1}} \ldots r\left(\varphi_{p}\right)^{m_{p}}
$$

The next steps of the computation of the two-point correlation form factor are almost the same as above. The only difference with the periodic case is the necessity to know the density of periodic orbits, $\rho(l, \varphi)$, with fixed length and angle. Like in (50) one gets

$$
\rho(l, \varphi)=\frac{l}{4 \mathcal{A}} .
$$

In the semi-classical limit $k \rightarrow \infty$ under the assumption that the ratio $\left(x_{0} a\right) /\left(y_{0} b\right)$ is an irrational number the factors $\phi_{1}$ and $\phi_{2}$ in (71) and (72) are equivalent to independent random variables uniformly distributed between 0 and $\frac{1}{2} \pi$. It means that

$$
<f>=\lim _{k \rightarrow \infty} \frac{2}{\pi} \int_{0}^{\pi / 2} f\left(\phi_{1}(\varphi), \phi_{2}(\varphi)\right) d \varphi=\frac{4}{\pi^{2}} \int_{0}^{\pi / 2} \int_{0}^{\pi / 2} f\left(\phi_{1}, \phi_{2}\right) d \phi_{1} d \phi_{2}
$$


for any smooth function $f\left(\phi_{1}, \phi_{2}\right)$.

The integration over the angle $\varphi$ therefore gives for any integer $n$,

$$
<r(\varphi)^{n}>=\frac{4}{\pi^{2}} \int_{0}^{\pi / 2} \int_{0}^{\pi / 2} d \phi_{1} d \phi_{2} r^{n}\left(\phi_{1}, \phi_{2}\right),
$$

where we have set

$$
r\left(\phi_{1}, \phi_{2}\right)=4 \sin ^{2} \phi_{1} \sin ^{2} \phi_{2}
$$

Of course, the introduction of $r\left(\phi_{1}, \phi_{2}\right)$ is just a convenient way to perform the generalized diagonal approximation where one takes into account not only trajectories with exactly the same lengths but also ones which have equal lengths up to the first order expansion as in Eq. (69) (see [20]).

The only necessary integral is thus

$$
<r^{n}>=\frac{\left(C_{2 n}^{n}\right)^{2}}{4^{n}}=\left(\frac{(2 n) !}{2^{n}(n !)^{2}}\right)^{2} .
$$

The final expression for the 2-point correlation form factor of the rectangular billiard with Dirichlet boundary conditions is similar to Eq. (61)

$$
\begin{aligned}
K(\tau) & =\left\langle e^{-|\mathcal{D}|^{2} g r \tau / 16}\right\rangle \\
& +\sum_{p=2}^{\infty} \frac{1}{p !} \sum_{m, n \geq 0} A_{m n p}\left(\frac{-i g \mathcal{D}}{8}\right)^{m+p}\left(\frac{i g \overline{\mathcal{D}}}{8}\right)^{n+p} \tau^{m+n+p+1}
\end{aligned}
$$

where

$$
A_{m n p}=\frac{(m+p-1) !(n+p-1) !}{(m+n+p-1) !} \sum_{m_{i}, n_{j} \geq 0} \prod_{i=1}^{p}\left[\frac{C_{m_{i}+n_{i}}^{m_{i}}<r^{m_{i}+n_{i}+2}>}{\left(m_{i}+1\right) !\left(n_{i}+1\right) !}\right] .
$$

The sum here is taken as before over all non-negative $m_{i}$ and $n_{j}$ verifying $m_{1}+\cdots+m_{p}=m$ and $\left.n_{1}+\cdots+n_{p}=n\right)$ and the mean value $<\ldots>$ is evaluated in (83). Note that only the value of $A_{m n p}$ differs between the periodic case (61) and Eq. (84); and if $r$ is set equal to 1, (85) reduces to (62). In Appendix it is demonstrated that this expansion agrees with the exact results obtained in Ref. [1]. 


\section{Non-constant diffraction coefficient}

In the previous Sections we have assumed that the diffraction coefficient that appears in the expression of the density of diffractive orbits (12) is a constant (14) for a point-like scatter. In this Section we consider the general case when this diffraction coefficient depends on scattering angles.

Let a trajectory hit the singularity with momentum in the direction $\vec{n}$ and leave it with momentum in the direction $\vec{n}^{\prime}$. The diffraction coefficient $\mathcal{D}\left(\vec{n}, \vec{n}^{\prime}\right)$ is a certain function obeying the optical theorem (13).

\subsection{Periodic boundary conditions}

Trajectories with equal length play a very important role in the computation of the form factor. In the rectangular billiard with periodic boundary conditions the lengths of periodic and diffractive orbits are given by

$$
l=\sqrt{(M a)^{2}+(N b)^{2}}
$$

and orbits with $M, N$ of different signs are degenerated though geometrically they are different. When the diffraction coefficient is a constant all such trajectories give the same contribution but when it depends on initial and final directions their contribution will be different.

In the rectangular billiard orbits that hit or leave the singularity with any of the 4 angles

$$
g_{\alpha}(\varphi)=\varphi,-\varphi,-\pi+\varphi, \pi-\varphi
$$

all have the same lengths (see Fig. 6). Let us assume that $0 \leq \varphi<\pi / 2$ and label these angles respectively by $\alpha=1,2,3$ and 4 . To take into account trajectories with equal length it is convenient to label them by 2 quantities $(\varphi, \alpha)$ where the angle $\varphi$ is uniquely related with the length of the trajectory and belongs to the upper-right quadrant, $0 \leq \varphi<\pi / 2$, and the index $\alpha=1,2,3,4$ describes the form of the trajectory and determines which of the 4 angles (87) is the geometrical angle between the trajectory and the horizontal.

Let us denote by $D_{\alpha \beta}\left(\varphi, \varphi^{\prime}\right)$ the diffraction coefficient $\mathcal{D}\left(g_{\alpha}(\varphi), g_{\beta}\left(\varphi^{\prime}\right)\right)$ corresponding to a scattering process where the trajectory arrives with angle 


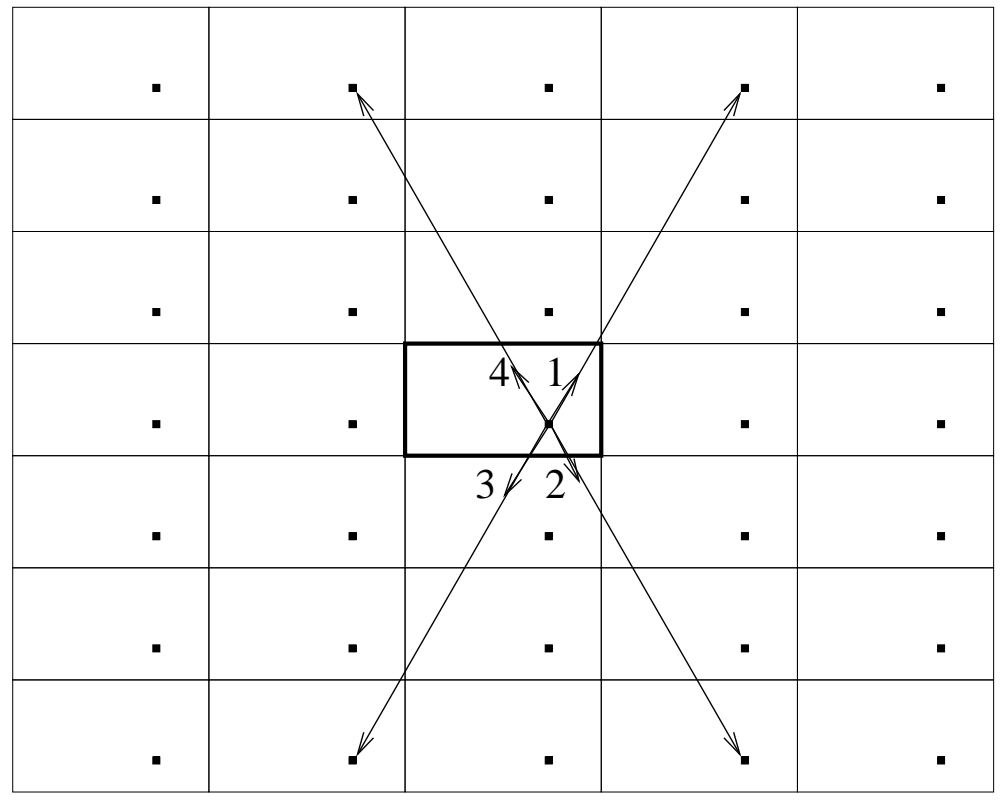

Figure 6: Four trajectories of same length in the rectangle with periodic boundary conditions

opposite to $(\varphi, \alpha)$ and leaves the scatter with angle $\left(\varphi^{\prime}, \beta\right)$. The explicit form of the matrix $D\left(\varphi, \varphi^{\prime}\right)$ is the following

$$
\begin{gathered}
\quad D\left(\varphi, \varphi^{\prime}\right)= \\
\left.\begin{array}{llll}
\mathcal{D}\left(\varphi, \varphi^{\prime}\right) & \mathcal{D}\left(\varphi,-\varphi^{\prime}\right) & \mathcal{D}\left(\varphi,-\pi+\varphi^{\prime}\right) & \mathcal{D}\left(\varphi, \pi-\varphi^{\prime}\right) \\
\mathcal{D}\left(-\varphi, \varphi^{\prime}\right) & \mathcal{D}\left(-\varphi,-\varphi^{\prime}\right) & \mathcal{D}\left(-\varphi,-\pi+\varphi^{\prime}\right) & \mathcal{D}\left(-\varphi, \pi-\varphi^{\prime}\right) \\
\mathcal{D}\left(-\pi+\varphi, \varphi^{\prime}\right) & \mathcal{D}\left(-\pi+\varphi,-\varphi^{\prime}\right) & \mathcal{D}\left(-\pi+\varphi,-\pi+\varphi^{\prime}\right) & \mathcal{D}\left(-\pi+\varphi, \pi-\varphi^{\prime}\right) \\
\mathcal{D}\left(\pi-\varphi, \varphi^{\prime}\right) & \mathcal{D}\left(\pi-\varphi,-\varphi^{\prime}\right) & \mathcal{D}\left(\pi-\varphi,-\pi+\varphi^{\prime}\right) & \mathcal{D}\left(\pi-\varphi, \pi-\varphi^{\prime}\right)
\end{array}\right)
\end{gathered}
$$

For instance (see Fig. 7) if the trajectory leaves the scatter with an angle $-\varphi$ (which in our notation is angle 2), it will come back with an angle $\pi-\varphi$, so it hits the singularity with an angle opposite to $\alpha=2$. If it leaves again with an angle, say $\varphi$ (angle 1), then the scattering amplitude between the incoming and the outgoing trajectory will be proportional to $\mathcal{D}(-\varphi, \varphi)$, which is just $D_{21}(\varphi, \varphi)$. The advantage of the matrix representation of the diffraction coefficient is that different variables $\varphi$ describe orbits with different lengths. 


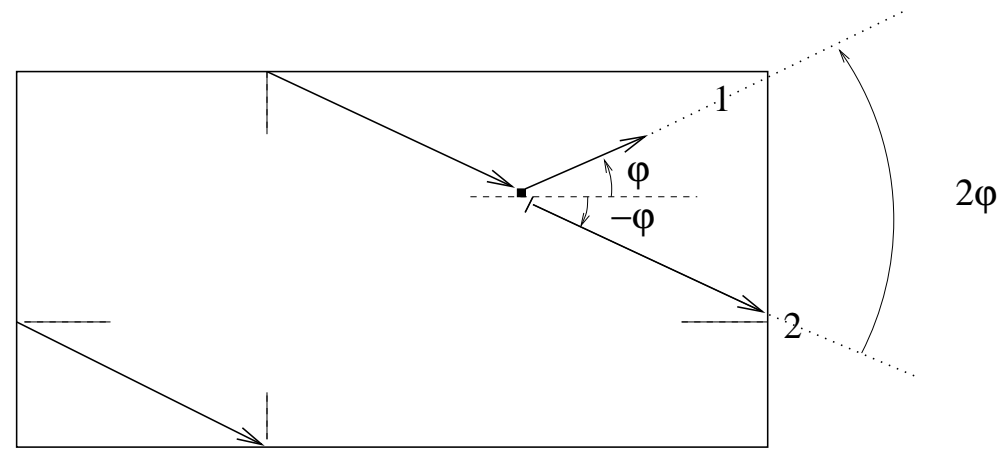

The scattering amplitude is $\mathrm{D}_{21}$

Figure 7: A periodic trajectory in the rectangle with periodic boundary conditions

The proliferation of diffractive orbits with the same lengths is taken into account automatically by matrix multiplication.

We now consider a trajectory made of several periodic orbits. Each time the trajectory leaves the scatter with angle $\alpha, 1 \leq \alpha \leq 4$, it comes back on the scatter with the unique angle corresponding to an additional phase $\pi$, then it leaves the scatter again with any angle $\beta, 1 \leq \beta \leq 4$. In the general formula (12) we sum over all initial and final vectors $\vec{n}_{i}, \vec{n}_{j}^{\prime}$ with the only condition that the trajectory is closed, which means that the outgoing angle of the first diffractive trajectory must correspond to the incoming angle of the last diffractive trajectory (more precisely they must differ by $\pi$ ). Suppose that we consider a multiple diffractive trajectory consisting of $m$ diffractive orbits beginning and ending at the singularity and such that all unfolded diffractive orbits are almost parallel to an angle $\varphi$ in the upper-right quadrant. Such trajectory will therefore contain a coefficient $D_{\alpha_{1} \alpha_{2}}(\varphi, \varphi) D_{\alpha_{2} \alpha_{3}}(\varphi, \varphi) \ldots D_{\alpha_{m} \alpha_{1}}(\varphi, \varphi)$. Since we have to take into account the degeneracy of the lengths and gather together all terms corresponding to trajectories of same total length $l_{1}+\cdots+l_{m}$, we have to sum over all possible 
incoming or outgoing angles:

$$
\sum_{\substack{1 \leq \alpha_{i} \leq 4 \\ 1 \leq i \leq m}} D_{\alpha_{1} \alpha_{2}}(\varphi, \varphi) D_{\alpha_{2} \alpha_{3}}(\varphi, \varphi) \ldots D_{\alpha_{m} \alpha_{1}}(\varphi, \varphi)=\operatorname{tr}\left(D^{m}(\varphi, \varphi)\right) .
$$

Note that all angles are equal as we consider trajectories with equal lengths. Of course, when $\mathcal{D}$ is a constant $\operatorname{tr}\left(D^{m}(\varphi, \varphi)\right)=(4 \mathcal{D})^{m}$ for all integer $m \geq 1$.

When the degeneracy has been taken into account the computation is the same as in Section 3 but $(g \mathcal{D})^{m}$ is replaced by $\operatorname{tr}\left(D^{m}(\varphi, \varphi)\right)$ for $m \geq 1$. The summation of such non-diagonal terms leads to the multiplication of the Green function contribution $G(L)$ (given by (33)) by an attenuation factor similar to the one in Eq. (32)

$$
\left[1+\operatorname{tr}\left(\exp \left(\frac{\rho D(\varphi, \varphi)}{2 i k} L\right)-1\right)\right] G(L),
$$

where as above $\rho=1 / \mathcal{A}$ is the density of diffraction points.

The higher-order terms in the density of states come from the contributions of higher-order saddle-point manifolds. Let us consider a composite trajectory consisting in $m$ diffractive orbits, among which $m_{1}$ are almost parallel to the direction $\varphi_{1}, m_{2}$ to the direction $\varphi_{2}$, and more generally $m_{i}$ are almost parallel to the direction $\varphi_{i}$ for $1 \leq i \leq p$. Each of such trajectories is a permutation of sequence (35) but contrary to the previous Section for each diffractive orbit there is an additional label $\alpha_{i}=1,2,3,4$ corresponding to one of the 4 angles (87).

The total number of different saddle-point trajectories for $p, m$ fixed and given $m_{i}$ and angle $\left(\varphi_{i}, \alpha_{i}\right), 1 \leq i \leq p$, is

$$
4^{m} \frac{m !}{m_{1} ! \ldots m_{p} !}
$$

the coefficient 4 taking into account the fact that each orbit is 4 times degenerate (we will always consider $0 \leq \varphi<\pi / 2$ ).

For instance at Fig. 8 there are 2 families of vectors, parallel to $\varphi_{1}=0$ or its images, or parallel to $\varphi_{2}=\pi / 4$ or its images. At this figure two diffractive orbits are represented: orbits $1,4,5,7,8$ are of type 1 and orbits $2,3,6$ are of type 2. But since now the scattering amplitude $\mathcal{D}$ is not a constant, the saddle-points will give different contributions depending on the geometrical form of diffractive orbits. 

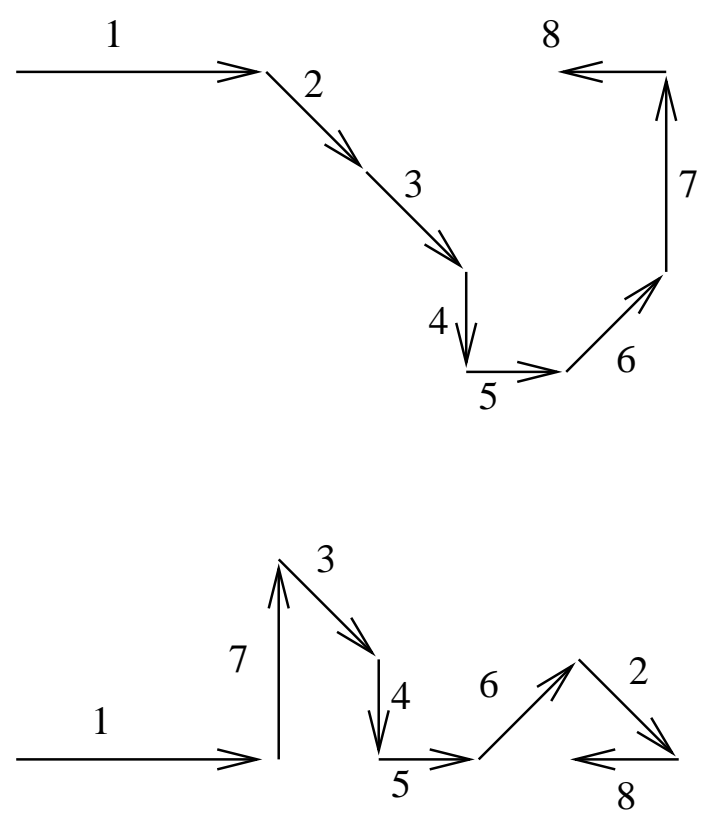

Figure 8: Diffractive trajectories for $m=8, p=2, m_{1}=5, m_{2}=3$ with the same length.

The $k$-th orbit is determined by a pair $\left(\varphi_{i_{k}}, \alpha_{k}\right)$ with $\alpha_{k}=1, \ldots, 4$ and $0 \leq \varphi_{i_{k}}<\pi / 2$.

For a trajectory with $p$ possible angles, $\varphi_{i_{k}}$ can take $p$ different values $\left\{\varphi_{1}, \ldots, \varphi_{p}\right\}$. Each diffraction on the scatter gives a coefficient which generic form is $D_{\alpha_{k}, \alpha_{k+1}}\left(\varphi_{i_{k}}, \varphi_{i_{k+1}}\right)$, since the coefficient $D_{i j}\left(\varphi, \varphi^{\prime}\right)$ is the scattering amplitude corresponding to a diffraction where an orbit leaves the scatter with the image of type $i$ of the angle $\varphi$, and comes back and leaves again with the image $j$ of the angle $\varphi^{\prime}$. In order to take into account all trajectories we have to sum over all $\alpha_{k}, 1 \leq \alpha_{k} \leq 4$, and all $i_{k}, 1 \leq i_{k} \leq p$, for $1 \leq k \leq m$ (of course due to the fact that all orbits are periodic, the index $i_{m+1}=i_{1}$ ).

Thus for fixed $m_{i}$ and $\varphi_{i}$ the sum over all possible scatterings of the 
scattering amplitudes gives

$$
R\left(\left(m_{1}, \varphi_{1}\right), \ldots,\left(m_{p}, \varphi_{p}\right)\right)=\sum_{p e r m} \operatorname{tr}\left(D\left(\varphi_{i_{1}}, \varphi_{i_{2}}\right) D\left(\varphi_{i_{2}}, \varphi_{i_{3}}\right) \ldots D\left(\varphi_{i_{m}}, \varphi_{i_{1}}\right)\right)
$$

where the summation is performed over all permutations $\left(i_{1}, \ldots, i_{m}\right)$ of the sequence (35).

E.g. for the considered example $m=4$ and $m_{1}=m_{2}=2$ (see Fig. 四b and (37))

$$
\begin{aligned}
& R\left(\left(2, \varphi_{1}\right),\left(2, \varphi_{2}\right)\right)=\operatorname{tr}\left(D\left(\varphi_{1}, \varphi_{1}\right) D\left(\varphi_{1}, \varphi_{2}\right) D\left(\varphi_{2}, \varphi_{2}\right) D\left(\varphi_{2}, \varphi_{1}\right)\right) \\
& +\operatorname{tr}\left(D\left(\varphi_{1}, \varphi_{2}\right) D\left(\varphi_{2}, \varphi_{1}\right) D\left(\varphi_{1}, \varphi_{2}\right) D\left(\varphi_{2}, \varphi_{1}\right)\right) \\
& +\operatorname{tr}\left(D\left(\varphi_{1}, \varphi_{2}\right) D\left(\varphi_{2}, \varphi_{2}\right) D\left(\varphi_{2}, \varphi_{1}\right) D\left(\varphi_{1}, \varphi_{1}\right)\right) \\
& +\operatorname{tr}\left(D\left(\varphi_{2}, \varphi_{2}\right) D\left(\varphi_{2}, \varphi_{1}\right) D\left(\varphi_{1}, \varphi_{1}\right) D\left(\varphi_{1}, \varphi_{2}\right)\right) \\
& +\operatorname{tr}\left(D\left(\varphi_{2}, \varphi_{1}\right) D\left(\varphi_{1}, \varphi_{2}\right) D\left(\varphi_{2}, \varphi_{1}\right) D\left(\varphi_{1}, \varphi_{2}\right)\right) \\
& +\operatorname{tr}\left(D\left(\varphi_{2}, \varphi_{1}\right) D\left(\varphi_{1}, \varphi_{1}\right) D\left(\varphi_{1}, \varphi_{2}\right) D\left(\varphi_{2}, \varphi_{2}\right)\right) .
\end{aligned}
$$

The quantity $R\left(\left(m_{1}, \varphi_{1}\right), \ldots,\left(m_{p}, \varphi_{p}\right)\right)$ replaces the corresponding quantity (42) in Eq. (41) and reduces to it if $\mathcal{D}$ is a constant.

The rest of the calculations is exactly the same as above and finally one gets the expression (45) where

$$
d_{1}(\vec{L})=\frac{i \mathcal{A}}{2 \pi}\left[1+\operatorname{tr}\left(\exp \left(\frac{D(\varphi, \varphi)}{2 i k \mathcal{A}} L\right)-1\right)\right]
$$

and $d_{p}\left(\vec{L}_{1}, \ldots, \vec{L}_{p}\right)$ is the same as (41) with $R\left(m_{1}, \ldots, m_{p}\right)$ replaced by $R$ given by (92).

Using Eq. (60) and replacing (42) by (92) we obtain that the two-point correlation form factor for the considered case is given by the following formulas

$$
\begin{aligned}
K(\tau) & =<\left|1+\operatorname{tr}\left(\exp \left(-i \frac{D(\varphi, \varphi)}{8} \tau\right)-1\right)\right|^{2}> \\
& +\sum_{p=2}^{\infty} \frac{1}{p !} \sum_{m, n \geq p} A_{m n p} \tau^{m+n-p+1}
\end{aligned}
$$


with

$$
\begin{aligned}
& A_{m n p}=\frac{(-i / 8)^{m}(i / 8)^{n}}{m n(m+n-p-1) !} \sum_{\substack{\sum_{i}=m \\
m_{i} \geq 1}} \sum_{\substack{\sum_{\begin{subarray}{c}{j \\
n_{j} \geq 1} }}=n} \\
{n_{j} \geq 1}\end{subarray}}^{p}\left[\frac{\left(m_{i}+n_{i}-2\right) !}{\left(m_{i}-1\right) !\left(n_{i}-1\right) !}\right] \\
& \times<R\left(\left(m_{1}, \varphi_{1}\right), \ldots,\left(m_{p}, \varphi_{p}\right)\right) \bar{R}\left(\left(n_{1}, \varphi_{1}\right), \ldots,\left(n_{p}, \varphi_{p}\right)\right)>,
\end{aligned}
$$

where $R\left(\left(m_{1}, \varphi_{1}\right), \ldots,\left(m_{p}, \varphi_{p}\right)\right)$ is defined in Eq. (92). The sum is taken over all partitions of $m$ and $n$ into sums of integers $m_{i}$ and $n_{j}$ greater than 1 verifying $m_{1}+\cdots+m_{p}=m$ and $n_{1}+\cdots+n_{p}=n$.

The symbol $\langle\ldots\rangle$ means the integration over all indicated angle variables from 0 to $\pi / 2$

$$
<f\left(\varphi_{1}, \ldots, \varphi_{k}\right)>=\left(\frac{2}{\pi}\right)^{k} \int_{0}^{\pi / 2} d \varphi_{1} \ldots \int_{0}^{\pi / 2} d \varphi_{k} f\left(\varphi_{1}, \ldots, \varphi_{k}\right) .
$$

\subsection{Dirichlet boundary conditions}

Dirichlet boundary conditions give rise to a slight complication as there are now 16 diffractive orbits associated with each couple of positive integers $(M, N)$ (see Fig. 9). In Eq. (12) the contribution of a multiple diffractive trajectory consisting in $m$ diffractive orbits beginning and ending at the singularity and parallel to a fixed direction $\varphi$ contains products of diffraction coefficients $\mathcal{D}\left(\varphi_{i}, \varphi_{j}\right)$ and terms $\exp \left(i k l_{i}\right)$ coming from the Green function where $l_{i}$ is the length of one of the 16 orbits. If we expand $l_{i}$ for large $M, N$ each term $\exp \left(i k l_{i}\right)$ can be written according to (69) as

$$
\epsilon_{1} \epsilon_{2} e^{i k l_{i}-i \frac{\pi}{2} \nu_{i}}=e^{i k l_{p}} e^{i\left(\epsilon_{1}-1\right) k x_{0} \cos \varphi+i\left(\epsilon_{2}-1\right) k y_{0} \sin \varphi}
$$

where $l_{p}$ is the length (67) of the closest periodic orbit and $\epsilon_{1}, \epsilon_{2}= \pm 1$. As in the previous Section we will denote respectively by $1,2,3$, and 4 the angles $\varphi,-\varphi,-\pi+\varphi$ and $\pi-\varphi$ corresponding to the symmetry group of the rectangle.

For the periodic case (see Fig. 6) a trajectory which leaves the scatter with angle $(\varphi, \alpha)$ comes back to it with an angle which is necessarily the angle opposite to the angle $\alpha$. Therefore for periodic boundary conditions the free motion between scatters is fixed and the proliferation of diffractive orbits only comes from the scattering process. 


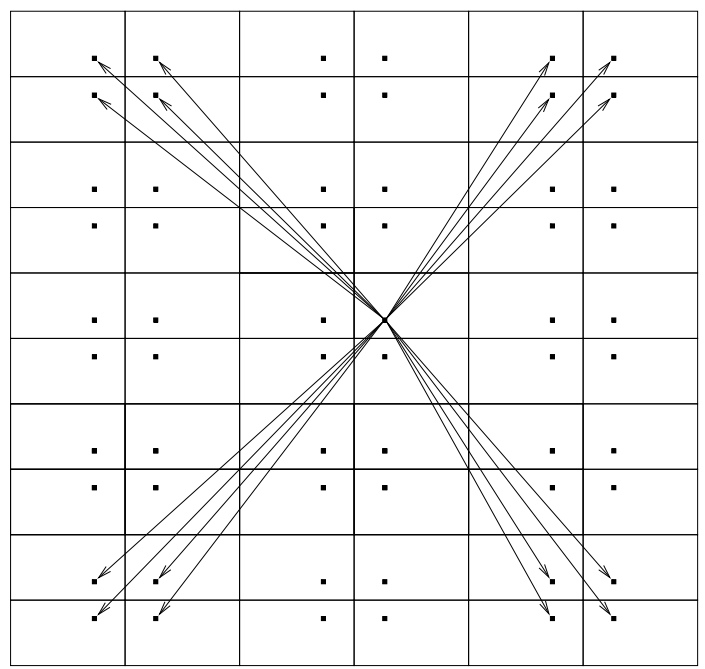

Figure 9: The 16 trajectories of almost same length in the rectangle with Dirichlet boundary conditions.

For the Dirichlet boundary conditions (see Fig. 9) by small changing of the initial angle the trajectory $(\varphi, \alpha)$ can come back to the scatter with any of the 4 angles (87) corresponding to $\varphi$, which leads to another source of diffractive orbits with almost the same length.

Let us denote by $T_{\alpha \beta}(\varphi)$ the coefficient corresponding to a diffractive orbit leaving the diffractive center with an angle $(\varphi, \alpha)$ and coming back with angle opposite to $(\varphi, \beta)$. Each $T_{\alpha \beta}(\varphi)$ is a product of two factors of the type

$$
\epsilon_{1} \epsilon_{2} \exp \left[i\left(\epsilon_{1}-1\right) \phi_{1}+i\left(\epsilon_{2}-1\right) \phi_{2}\right]
$$

where angles $\phi_{i}=\phi_{i}(\varphi)$ were defined in (71) and (72), and $\epsilon_{i}= \pm 1$. The matrix $T=T(\varphi)$ describes the free motion modification of the Green function and simple calculations show that it has the following form

$$
T(\varphi)=\left(\begin{array}{cccc}
1 & \bar{b} & \bar{a} \bar{b} & \bar{a} \\
b & 1 & \bar{a} & b \bar{a} \\
a b & a & 1 & b \\
a & a \bar{b} & \bar{b} & 1
\end{array}\right)
$$


where $a=-e^{2 i \phi_{1}}$ and $b=-e^{2 i \phi_{2}}$.

It can be checked that

$$
T_{\alpha \beta}(\varphi)=V_{\alpha}(\varphi) \bar{V}_{\beta}(\varphi)
$$

where $V_{i}\left(\phi_{1}(\varphi), \phi_{2}(\varphi)\right)$ is the vector defined by

$$
V(\vec{\phi})=\left(\begin{array}{c}
\exp \left(-i \phi_{1}-i \phi_{2}\right) \\
-\exp \left(-i \phi_{1}+i \phi_{2}\right) \\
\exp \left(i \phi_{1}+i \phi_{2}\right) \\
-\exp \left(i \phi_{1}-i \phi_{2}\right)
\end{array}\right)
$$

Any diffractive trajectory can be specified by fixing initial and final angles for each scattering. If the trajectory leaves the scatter with an angle $(\varphi, k)$ and comes back to it with angle $(\varphi, l+\pi)$ (that is, an angle opposite to $l)$ it is attributed a coefficient $T_{k l}(\varphi)$ (100). If then it scatters to an angle $\left(\varphi^{\prime}, j\right)$ it gets the coefficient $D_{l j}\left(\varphi, \varphi^{\prime}\right)$ defined in (\$8) and so on (see Fig. 19). For

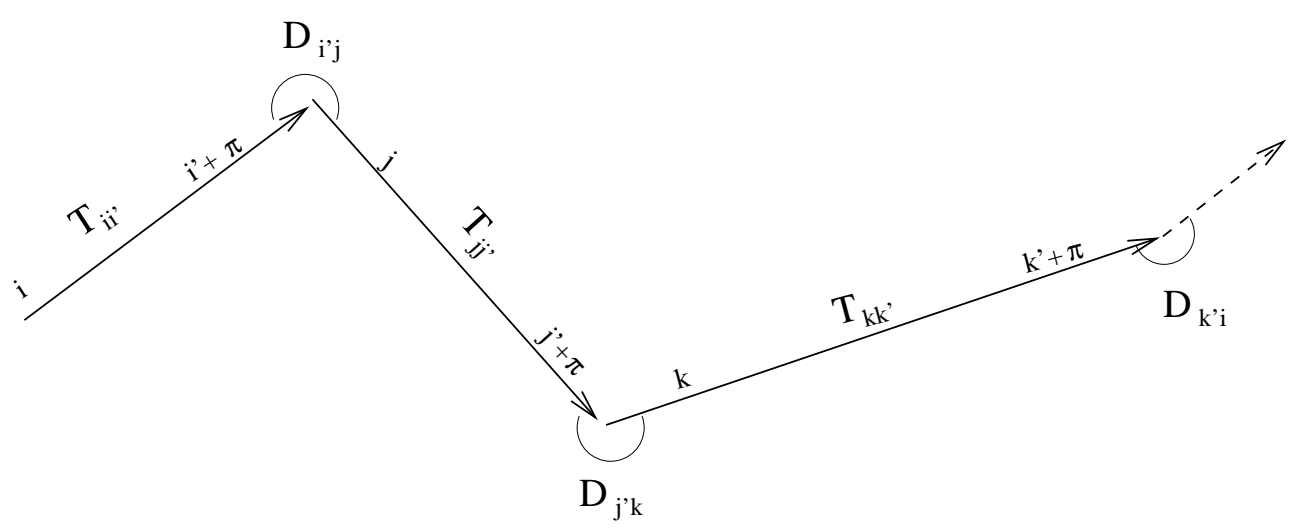

Figure 10: An example of periodic trajectory for $m=3$

example, a diffractive trajectory consisting in $m$ diffractive orbits parallel to the direction $\varphi$ will be associated to a pre-factor

$$
\sum T_{\alpha_{1} \alpha_{1}^{\prime}} D_{\alpha_{1}^{\prime} \alpha_{2}} T_{\alpha_{2} \alpha_{2}^{\prime}} \ldots T_{\alpha_{m} \alpha_{m}^{\prime}} D_{\alpha_{m}^{\prime} \alpha_{1}}=\operatorname{tr}(T D)^{m} .
$$


As in Section 4 assuming that the ratio $\left(x_{0} a\right) /\left(y_{0} b\right)$ is an irrational number then as $k \rightarrow \infty$ angles $\phi_{1}$ and $\phi_{2}$ act as independent random variables uniformly distributed between 0 and $\pi / 2$.

To take into account higher-order contributions we have to sum over all possible types of vectors $\left(\alpha_{i}, \alpha_{j}^{\prime}=1\right.$ to 4$)$ and all possible angles $\varphi_{i_{k}} \in$ $\left\{\varphi_{1}, \ldots, \varphi_{p}\right\}$ in terms like

$$
\begin{array}{r}
\sum_{\alpha_{i}, \alpha_{j}^{\prime}} D_{\alpha_{1}, \alpha_{1}^{\prime}}\left(\varphi_{i_{1}}, \varphi_{i_{2}}\right) T_{\alpha_{1}^{\prime}, \alpha_{2}}\left(\varphi_{i_{2}}\right) D_{\alpha_{2}, \alpha_{2}^{\prime}}\left(\varphi_{i_{2}}, \varphi_{i_{3}}\right) T_{\alpha_{2}^{\prime}, \alpha_{3}}\left(\varphi_{i_{3}}\right) \ldots \\
=\operatorname{tr}\left(D\left(\varphi_{i_{1}}, \varphi_{i_{2}}\right) T\left(\varphi_{i_{2}}\right) D\left(\varphi_{i_{2}}, \varphi_{i_{3}}\right) T\left(\varphi_{i_{3}}\right) \ldots\right)
\end{array}
$$

under the constraint that each angle $\varphi_{i}$ appears exactly $m_{i}$ times. Using the property (101) one can rewrite the above expression by introducing the effective diffraction coefficient $S$ defined by

$$
S\left(\varphi, \varphi^{\prime}\right)={ }^{t} \bar{V}_{\alpha}(\varphi) D_{\alpha \beta}\left(\varphi, \varphi^{\prime}\right) V_{\beta}\left(\varphi^{\prime}\right)
$$

which describes all possibilities of scattering and free motion between the scatter. Since $S\left(\varphi, \varphi^{\prime}\right)$ is a number we get

$$
\begin{aligned}
& \operatorname{tr}\left(D\left(\varphi_{i_{1}}, \varphi_{i_{2}}\right) T\left(\varphi_{i_{2}}\right) D\left(\varphi_{i_{2}}, \varphi_{i_{3}}\right) T\left(\varphi_{i_{3}}\right) \ldots\right) \\
& \quad=S\left(\varphi_{i_{1}}, \varphi_{i_{2}}\right) S\left(\varphi_{i_{2}}, \varphi_{i_{3}}\right) \ldots S\left(\varphi_{i_{m}}, \varphi_{i_{1}}\right)
\end{aligned}
$$

Let us define a quantity similar to Eq. (92)

$$
R\left(\left(m_{1}, \varphi_{1}\right), \ldots,\left(m_{p}, \varphi_{p}\right)\right)=\sum_{p e r m} S\left(\varphi_{i_{1}}, \varphi_{i_{2}}\right) S\left(\varphi_{i_{2}}, \varphi_{i_{3}}\right) \ldots S\left(\varphi_{i_{m}}, \varphi_{i_{1}}\right),
$$

where the sum is taken over all permutations $\left(i_{1}, \ldots, i_{m}\right)$ of the sequence (35) and it is implicitly assumed the set $\varphi_{i}$ contains $m_{1}$ terms of $\varphi_{1}, m_{2}$ terms of $\varphi_{2}, \ldots, m_{p}$ terms of $\varphi_{p}$. It can be checked that Eq. (107) reduces to (78) if $\mathcal{D}$ is a constant, since we have

$$
\sum_{i, j}{ }^{t} \bar{V}_{i}(\varphi) V_{j}(\varphi)=\operatorname{gr}(\varphi)
$$

with $g=4$ and $r(\varphi)$ given by (74). The final expression for the two-point correlation form factor, $K(\tau)$, follows from exactly the same consideration as 
in previous Sections. It reads

$$
\begin{aligned}
K(\tau)=< & <\left|\exp \left(-i \frac{S(\varphi, \varphi)}{8} \tau\right)\right|^{2}>> \\
& +\sum_{p=2}^{\infty} \frac{1}{p !} \sum_{m, n \geq p} A_{m n p} \tau^{m+n-p+1}
\end{aligned}
$$

where

$$
\begin{aligned}
A_{m n p} & =\frac{(-i / 8)^{m}(i / 8)^{n}}{m n(m+n-p-1) !} \sum_{\substack{\sum_{m_{i}=m} \\
m_{i} \geq 1}} \sum_{\substack{\sum_{n} n_{j}=n \\
n_{j} \geq 1}} \prod_{i=1}^{p}\left[\frac{\left(m_{i}+n_{i}-2\right) !}{\left(m_{i}-1\right) !\left(n_{i}-1\right) !}\right] \\
& \times<<R\left(\left(m_{1}, \varphi_{1}\right), \ldots,\left(m_{p}, \varphi_{p}\right)\right) \bar{R}\left(\left(n_{1}, \varphi_{1}\right), \ldots,\left(n_{p}, \varphi_{p}\right)>>\right.
\end{aligned}
$$

As usual the sum is taken over all partitions of $m$ and $n$ into sums of $p$ positive integers. The symbol $\langle<\ldots>>$ denotes the double average over random phases $\phi_{i}$ as in Eq. (81) and geometrical angles $\varphi_{j}$ as in Eq. (97).

\section{Conclusion}

We have discussed the spectral statistics of a rectangular billiard with a small-size impurity inside and developed the method which permits the explicit construction of perturbation expansion of the two-point correlation form factor for this system, $K(\tau)$, into series of $\tau$

$$
K(\tau)=\sum_{n} c_{n} \tau^{n}
$$

Using the method of Ref. [20] we demonstrate that after the summation over diffractive orbits with approximately the same lengths the oscillating part of the density of states can be written in the form (45)

$$
d^{(o s c)}(E)=\sum_{p=1}^{\infty} \sum_{L_{1}<\ldots<L_{p}} d_{p}\left(\vec{L}_{1}, \ldots, \vec{L}_{p}\right) G\left(L_{1}\right) \ldots G\left(L_{p}\right)+c . c .
$$


with

$$
\begin{aligned}
& d_{p}\left(\vec{L}_{1}, \ldots, \vec{L}_{p}\right)=\frac{L_{1}+\ldots+L_{p}}{4 \pi k} \sum_{m=p}^{\infty} \frac{1}{m} \\
& \times \sum_{\substack{m_{1}+\ldots+m_{p}=m \\
m_{i} \geq 1}} R\left(\left(m_{1}, \varphi_{1}\right), \ldots,\left(m_{p}, \varphi_{p}\right)\right) \\
& \times\left(\frac{L_{1}}{2 i k \mathcal{A}}\right)^{m_{1}-1} \frac{1}{\left(m_{1}-1\right) !} \ldots\left(\frac{L_{p}}{2 i k \mathcal{A}}\right)^{m_{p}-1} \frac{1}{\left(m_{p}-1\right) !}
\end{aligned}
$$

where the summation is taken over all partitions of $m$ into sums of $p$ positive integers $m=m_{1}+\ldots+m_{p}$ and $R\left(\left(m_{1}, \varphi_{1}\right), \ldots,\left(m_{p}, \varphi_{p}\right)\right)$ (107) is the contribution of all diagrams describing the scattering process of composite diffractive orbits consisting of $m_{1}$ orbits of type $1, m_{2}$ orbits of type 2 , and so on.

To construct such diagrams it is convenient first to write down all permutations of the sequence (35) of $m$ elements with $m_{i}$ elements of type $i$. The total number of these permutations is

$$
N\left(m_{1}, \ldots m_{p}\right)=\frac{m !}{m_{1} ! \ldots m_{p} !} .
$$

Each symbol $i$ represents the angle $\varphi_{i}$ and the constructed sequence of symbols is considered as the representation of the scattering process. The symmetry group of the rectangular billiard leads to the necessity to consider together all symmetry partners of a given trajectory, that is all possible choices for each diffractive orbit.

For periodic boundary conditions there are 4 different possibilities (87) as indicated at Fig. 6, whereas for Dirichlet conditions it gives rise to 16 trajectories with approximately the same lengths as at Fig. 9. To take into account all these degenerate and almost degenerate trajectories it is useful to use the matrix formalism developed in the previous Sections. All the scattering processes between 2 consecutive angles $\varphi$ and $\varphi^{\prime}$ are described by the matrix $D\left(\varphi, \varphi^{\prime}\right)$ defined in Eq. (88) for periodic boundary conditions, and by the quantity $S\left(\varphi, \varphi^{\prime}\right)$ defined in Eq. (105) for Dirichlet ones.

The quantity $R\left(\left(m_{1}, \varphi_{1}\right), \ldots,\left(m_{p}, \varphi_{p}\right)\right)$ is the sum of such contributions for all permutations of our standard sequence (35), that is all possible orderings of the sequence of diffractive orbits. 
The knowledge of $R\left(\left(m_{1}, \varphi_{1}\right), \ldots,\left(m_{p}, \varphi_{p}\right)\right)$ permits easily the computation of the two-point correlation form factor in all cases. The advantage of the representation (112) is that different terms in it are non-coherent and the two-point correlation function is simply given by the sum of the squares of each terms as in Eq. (49). This 'generalized diagonal approximation' leads to explicit formulas for the form factor Eqs. (95), (96) for the periodic boundary conditions and Eqs. (109) and (110) for the Dirichlet ones which solve the problem of the summation over non-diagonal terms for integrable systems.

\section{Appendix}

The exact expression for the two-point correlation function, $R_{2}(\epsilon)$, for a rectangular billiard perturbed by a small-size impurity with constant diffraction coefficient has been derived in Ref. [1]. The aim of this Appendix is to obtain its perturbation expansion for large $\epsilon$ and the corresponding expansion of the two-point correlation form factor, $K(\tau)$, into series of $\tau$.

According to Eq. (143) in [1] (with slight changing of the notations) the two-point correlation function can be written as multiple integrals

$$
\begin{aligned}
& R_{2}(\epsilon)-1=\int_{0}^{\infty} d \alpha_{1} \int_{0}^{\infty} d \alpha_{2} e^{-\pi \epsilon<J\left(r \alpha_{1}, r \alpha_{2}\right)>} \\
& \times\left[<r J_{0}\left(2 r \sqrt{\alpha_{1} \alpha_{2}}\right) e^{i r\left(\alpha_{1}+\alpha_{2}\right)}>^{2}+<r J_{1}\left(2 r \sqrt{\alpha_{1} \alpha_{2}}\right) e^{i r\left(\alpha_{1}+\alpha_{2}\right)}>^{2}\right]+\text { c.c. }
\end{aligned}
$$

where

$$
\begin{gathered}
J\left(\alpha_{1}, \alpha_{2}\right)=\left(\alpha_{1}-\alpha_{2}\right)\left(1+\frac{i}{\pi v^{\prime}}+2 i e^{i \alpha_{2}} \int_{\alpha_{1}}^{\infty} J_{0}\left(2 \sqrt{\alpha_{2} t}\right) e^{i t} d t\right) \\
-i\left[\alpha_{1} J_{0}\left(2 \sqrt{\alpha_{1} \alpha_{2}}\right)+\sqrt{\alpha_{1} \alpha_{2}} J_{1}\left(2 \sqrt{\alpha_{1} \alpha_{2}}\right)\right] e^{i\left(\alpha_{1}+\alpha_{2}\right)}
\end{gathered}
$$

Here $J_{0}(x)$ and $J_{1}(x)$ are the usual Bessel functions. The renormalized coupling constant $v^{\prime}$ is connected with the diffraction coefficient, $\mathcal{D}$, by the relation

$$
\mathcal{D}=\frac{1}{\frac{i}{4}+\frac{1}{4 \pi v^{\prime}}} .
$$


The variable $r=r\left(\phi_{1}, \phi_{2}\right)$ depends on the boundary conditions (see [1])

$$
r\left(\phi_{1}, \phi_{2}\right)=\left\{\begin{array}{cl}
1 & \text { for periodic boundary conditions } \\
4 \sin ^{2}\left(\phi_{1}\right) \sin ^{2}\left(\phi_{2}\right) & \text { for Dirichlet boundary conditions } \\
4 \cos ^{2}\left(\phi_{1}\right) \cos ^{2}\left(\phi_{2}\right) & \text { for Neumann boundary conditions }
\end{array},\right.
$$

where $\phi_{1}$ and $\phi_{2}$ are independent angles distributed uniformly between 0 and $\pi / 2$. The symbol $\langle\ldots\rangle$ denotes the mean values over these angles

$$
<f\left(\phi_{1}, \phi_{2}>=\frac{4}{\pi^{2}} \int_{0}^{\pi / 2} d \phi_{1} \int_{0}^{\pi / 2} d \phi_{2} f\left(\phi_{1}, \phi_{2}\right) .\right.
$$

In particular we have $\langle r(\phi, \phi)\rangle=1$. Changing variables $\epsilon$ to $x$ and $\alpha_{1}, \alpha_{2}$ to $\alpha_{1} / x, \alpha_{2} / x$, one gets

$$
\begin{aligned}
& R_{2}(x)-1=\int_{0}^{\infty} d \alpha_{1} \int_{0}^{\infty} d \alpha_{2} e^{-\pi x<J\left(\frac{r \alpha_{1}}{x}, \frac{r \alpha_{2}}{x}\right)>} \\
& \times\left[<r J_{0}\left(\frac{2 r \sqrt{\alpha_{1} \alpha_{2}}}{x}\right) e^{i r \frac{\alpha_{1}+\alpha_{2}}{x}}>^{2}+<r J_{1}\left(\frac{2 r \sqrt{\alpha_{1} \alpha_{2}}}{x}\right) e^{i r \frac{\alpha_{1}+\alpha_{2}}{x}}>^{2}\right]+\text { c.c. }
\end{aligned}
$$

To compute the expansion of the above expressions into powers of $1 / x$ it is convenient to use the transformations proposed in [27.

From (116) it follows that

$$
\left(\frac{\partial}{\partial \alpha_{1}}+\frac{\partial}{\partial \alpha_{2}}\right) x J\left(\frac{\alpha_{1}}{x}, \frac{\alpha_{2}}{x}\right)=2 J_{0}\left(\frac{2 \sqrt{\alpha_{1} \alpha_{2}}}{x}\right) e^{i \frac{\alpha_{1}+\alpha_{2}}{x}}
$$

and

$$
\frac{\partial}{\partial x} x J\left(\frac{\alpha_{1}}{x}, \frac{\alpha_{2}}{x}\right)=-2 i \frac{\sqrt{\alpha_{1} \alpha_{2}}}{x} J_{1}\left(\frac{2 \sqrt{\alpha_{1} \alpha_{2}}}{x}\right) e^{i \frac{\alpha_{1}+\alpha_{2}}{x}} .
$$

Using the fact that $J_{0}^{\prime}(x)=-J_{1}(x)$ and $J_{1}^{\prime}(x)=J_{0}(x)-J_{1}(x) / x$, one can check that

$$
\left(\frac{\partial}{\partial \alpha_{1}}+\frac{\partial}{\partial \alpha_{2}}\right)^{2} x J\left(\frac{\alpha_{1}}{x}, \frac{\alpha_{2}}{x}\right)=\frac{x^{2}}{\alpha_{1} \alpha_{2}} \frac{\partial^{2}}{\partial x^{2}} x J\left(\frac{\alpha_{1}}{x}, \frac{\alpha_{2}}{x}\right) .
$$


Therefore,

$$
\begin{aligned}
& {\left[\left(\frac{\partial}{\partial \alpha_{1}}+\frac{\partial}{\partial \alpha_{2}}\right)^{2}-\frac{x^{2}}{\alpha_{1} \alpha_{2}} \frac{\partial^{2}}{\partial x^{2}}\right] e^{-\pi x<J\left(\frac{r \alpha_{1}}{x}, \frac{r \alpha_{2}}{x}\right)>}=} \\
& \pi^{2}\left[\left(\left(\frac{\partial}{\partial \alpha_{1}}+\frac{\partial}{\partial \alpha_{2}}\right) x<J\left(\frac{r \alpha_{1}}{x}, \frac{r \alpha_{2}}{x}\right)>\right)^{2}\right. \\
& \left.-\frac{x^{2}}{\alpha_{1} \alpha_{2}}\left(\frac{\partial}{\partial x} x<J\left(\frac{r \alpha_{1}}{x}, \frac{r \alpha_{2}}{x}\right)>\right)^{2}\right] e^{-\pi x<J\left(\frac{r \alpha_{1}}{x}, \frac{r \alpha_{2}}{x}\right)>}
\end{aligned}
$$

and according to Eqs. (121) and (122) the previous equation can be transform as follows

$$
\begin{aligned}
& \frac{1}{4 \pi^{2}}\left[\left(\frac{\partial}{\partial \alpha_{1}}+\frac{\partial}{\partial \alpha_{2}}\right)^{2}-\frac{x^{2}}{\alpha_{1} \alpha_{2}} \frac{\partial^{2}}{\partial x^{2}}\right] e^{-\pi x<J\left(\frac{r \alpha_{1}}{x}, \frac{r \alpha_{2}}{x}\right)>}= \\
& {\left[<r J_{0}(\zeta) e^{i r \frac{\alpha_{1}+\alpha_{2}}{x}}>^{2}+<r J_{1}(\zeta) e^{i r \frac{\alpha_{1}+\alpha_{2}}{x}}>^{2}\right] e^{-\pi x<J\left(\frac{r \alpha_{1}}{x}, \frac{r \alpha_{2}}{x}\right)>},}
\end{aligned}
$$

where $\zeta=2 r \sqrt{\alpha_{1} \alpha_{2}} / x$.

The right-hand side of this expression is the pre-factor which appears in Eq. (120) and, consequently, one can express the two-point correlation function in the form

$$
\begin{aligned}
R_{2}(x)-1 & =\int_{0}^{\infty} \frac{d \alpha_{1} d \alpha_{2}}{4 \pi^{2} x^{2}}\left[\left(\frac{\partial}{\partial \alpha_{1}}+\frac{\partial}{\partial \alpha_{2}}\right)^{2}-\frac{x^{2}}{\alpha_{1} \alpha_{2}} \frac{\partial^{2}}{\partial x^{2}}\right] e^{-\pi x<J\left(\frac{r \alpha_{1}}{x}, \frac{r \alpha_{2}}{x}\right)>} \\
& + \text { c.c. }
\end{aligned}
$$

The derivatives with respect to $\alpha_{1}$ and $\alpha_{2}$ can be computed by integration by parts. It gives

$$
\begin{aligned}
& \int_{0}^{\infty} d \alpha_{1} \frac{\partial}{\partial \alpha_{1}}\left(\frac{\partial}{\partial \alpha_{1}}+\frac{\partial}{\partial \alpha_{2}}\right) e^{-\pi x<J\left(\frac{r \alpha_{1}}{x}, \frac{r \alpha_{2}}{x}\right)>}= \\
& 2 \pi\left\langle r e^{i r \alpha_{2} / x}\right\rangle e^{-\pi \alpha_{2}\left(1-\frac{i}{\pi v^{\prime}}\right)}
\end{aligned}
$$

and

$$
\begin{gathered}
\int_{0}^{\infty} d \alpha_{2} \frac{\partial}{\partial \alpha_{2}}\left(\frac{\partial}{\partial \alpha_{1}}+\frac{\partial}{\partial \alpha_{2}}\right) e^{-\pi x<J\left(\frac{r \alpha_{1}}{x}, \frac{r \alpha_{2}}{x}\right)>}= \\
2 \pi\left\langle r e^{i r \alpha_{1} / x}\right\rangle e^{-\pi \alpha_{1}\left(1+\frac{i}{\pi v^{\prime}}\right)} .
\end{gathered}
$$


Therefore

$$
\begin{gathered}
R_{0}(x)=\int_{0}^{\infty} \frac{d \alpha_{1} d \alpha_{2}}{4 \pi^{2} x^{2}}\left(\frac{\partial}{\partial \alpha_{1}}+\frac{\partial}{\partial \alpha_{2}}\right)^{2} e^{-\pi x<J\left(\frac{r \alpha_{1}}{x}, \frac{r \alpha_{2}}{x}\right)>}+\text { c.c. }= \\
\frac{1}{\pi x}\left\langle\frac{i r(r+i \pi x)}{(r+i \pi x)^{2}-\left(\frac{x}{v^{\prime}}\right)^{2}}\right\rangle+\text { c.c. }
\end{gathered}
$$

We are interested in the Fourier transform

$$
K(\tau)=\int_{-\infty}^{\infty} d x\left(R_{2}(x)-1\right) e^{2 i \pi \tau x}
$$

of the two-point correlation function.

The term (129) gives the following contribution

$$
K_{0}(\tau)=<e^{2 i \pi \tau x_{+}}+e^{2 i \pi \tau x_{-}}>-2 .
$$

where

$$
x_{ \pm}=\frac{r}{ \pm \frac{1}{v^{\prime}}-i \pi}
$$

are the poles in (129). Using the definition (117) of $\mathcal{D}$, Eqs. (131) and (132) lead to

$$
K_{0}(\tau)=<e^{-\frac{i r \mathcal{D} g}{8} \tau}+e^{\frac{i r \overline{\mathcal{D}} g}{8} \tau}>-2,
$$

where we introduce $g=4$ to be consistent with notations in Section 3.

The other contributions come from the second derivatives with respect to $x$ in (126). Using expansion (112) of Ref. [1] one gets

$$
\begin{aligned}
x<J\left(\frac{r \alpha_{1}}{x}, \frac{r \alpha_{2}}{x}\right)> & =\alpha_{1}+\alpha_{2}+\frac{i}{\pi v^{\prime}}\left(\alpha_{1}-\alpha_{2}\right) \\
& -2 i \sum_{m, n=0}^{\infty} \frac{\left(i \alpha_{1}\right)^{m+1}\left(i \alpha_{2}\right)^{n+1}}{x^{m+n+1}} \frac{(m+n) !<r^{m+n+2}>}{m !(m+1) ! n !(n+1) !} .
\end{aligned}
$$

The expansion of the exponential of this quantity yields

$$
\begin{gathered}
e^{-\pi x<J\left(\frac{r \alpha_{1}}{x}, \frac{r \alpha_{2}}{x}\right)>}=e^{-\pi\left(\alpha_{1}+\alpha_{2}\right)} e^{-\frac{i}{v^{\prime}}\left(\alpha_{1}-\alpha_{2}\right)} \sum_{p=0}^{\infty} \frac{(2 i \pi)^{p}}{p !} \sum_{m, n \geq 0} \\
\sum_{\substack{m_{1}+\cdots+m_{p}=m \\
n_{1}+\cdots+n_{p}=n}} \prod_{i=1}^{p} \frac{C_{m_{i}+n_{i}}^{m_{i}}}{\left(m_{i}+1\right) !\left(n_{i}+1\right) !}<r^{m_{i}+n_{i}+2}>\frac{\left(i \alpha_{1}\right)^{m+p}\left(i \alpha_{2}\right)^{n+p}}{x^{m+n+p}} .
\end{gathered}
$$


As

$$
\int_{0}^{\infty} t^{\alpha} e^{-\sigma t} d t=\frac{\alpha !}{\sigma^{\alpha+1}}
$$

the integration over $\alpha_{i}$ gives the expansion of the two-point correlation function into series of $1 / x$

$$
R_{2}(x)-1=\sum_{p=0}^{\infty} R_{p}(x),
$$

where $R_{0}(x)$ is given by (129) and

$$
R_{p}(x)=-\frac{(2 i \pi)^{p}}{p !} \sum_{m, n \geq 0} \frac{(m+n+p+1) ! A_{m n p}}{4 \pi^{2} x^{m+n+p+2}}\left(-\frac{\mathcal{D} g}{16 \pi}\right)^{m+p}\left(\frac{\overline{\mathcal{D}} g}{16 \pi}\right)^{n+p}
$$

where

$$
A_{m n p}=\frac{(m+p-1) !(n+p-1) !}{(m+n+p-1) !} \sum_{m_{i}, n_{j} \geq 0} \prod_{i=1}^{p}\left[\frac{C_{m_{i}+n_{i}}^{m_{i}}<r^{m_{i}+n_{i}+2}>}{\left(m_{i}+1\right) !\left(n_{i}+1\right) !}\right] .
$$

The expression (139) for $A_{m n p}$ is the same as in Eq. (85).

Taking the Fourier transform (130) of (138), we get the corresponding expansion of the two-point correlation form factor. The term corresponding to $p=1$ can be transformed to the form

$$
K_{1}(\tau)=<\left(e^{-\frac{i r \mathcal{D} g}{8} \tau}-1\right)\left(e^{\frac{i r \overline{\mathcal{D}} g}{8} \tau}-1\right)>.
$$

The sum of the contributions (133) and (140) (plus a 1 coming from the $\delta$-function in $R_{2}(x)$ ) gives

$$
<e^{-\frac{i r \mathcal{D} g}{8} \tau} e^{\frac{i r \overline{\mathcal{D}} g}{8} \tau}>=<e^{-\frac{|\mathcal{D}|^{2} g r}{16} \tau}>.
$$

The Fourier transform of the sum (138) gives the terms for $p \geq 2$

$$
\sum_{p=2}^{\infty} \frac{1}{p !} \sum_{m, n \geq 0} A_{m n p}\left(-\frac{i \mathcal{D} g}{8}\right)^{m+p}\left(\frac{i \overline{\mathcal{D}} g}{8}\right)^{n+p} \tau^{m+n+p+1},
$$

which coincides with the result (84). 


\section{References}

[1] Bogomolny E, Gerland U and Schmit C 1999 Phys. Rev. E 591315

[2] Bohigas O, Giannoni M J and Schmit C 1984 Phys. Rev. Lett. 521

[3] Mehta M L 1990 Random Matrix Theory (Springer, New York)

[4] Berry M V and Tabor M 1977 Proc. Roy. Soc. Lond. 356375

[5] Bohigas O 1991 in Chaos and Quantum Mechanics Giannoni M J, Voros A and Zinn-Justin J eds., Les Houches Summer School Lectures LII, 1989 (North Holland, Amsterdam, 1991) p 87

[6] Andreev A V and Altshuler B L 1995 Phys. Rev. Lett. 75 902; Agam O, Altshuler B L and Andreev A V 1995 Phys. Rev. Lett. 754389

[7] Bogomolny E B and Keating J P 1996 Phys. Rev. Lett. 771472

[8] Bogomolny E B in New Directions in Quantum Chaos, Casati G, Guarneri I and Smilansky U eds., 'Enrico Fermi' International School of Physics, Course CXLIII, Varenna, 1999 (IOS Press, Amsterdam, Oxford, Tokyo, Washington, 2000) p 333

[9] Marklof J 1998 Commun. Math. Phys. 199169

[10] Bogomolny E B, Leboeuf P and Schmit C 2000 Phys. Rev. Lett. 852486

[11] Šeba P 1990 Phys. Rev. Lett. 641855

[12] Shigehara T and Cheon T 1996 Phys. Rev. E 541321

[13] Jonckheere T, Gremaud B and Delande D 1998 Phys. Rev. Lett. 812442

[14] Bogomolny E B, Giraud O and Schmit C 2001 to be published

[15] Shklovskii B I, Shapiro B, Sears B R, Lambrianides P and Shore H B 1993 Phys. Rev. B 4711487

[16] Vattay G, Wirzba A and Rosenqvist P E 1994 Phys. Rev. Lett. 732304

[17] Pavloff N and Schmit C 1995 Phys. Rev. Lett. 75 61; 75 3779(E) 
[18] Berry M V 1985 Proc. Roy. Soc. A 400229

[19] Hannay J H and Ozorio de Almeida A M 1984 J. Phys. A: Math. Gen. 173429

[20] Bogomolny E B 2000 Nonlinearity 13947

[21] Balian R and Bloch C 1972 Ann. Phys. (N.Y.) 6976

[22] Gutzwiller M C in Chaos and Quantum Mechanics, Giannoni M J, Voros A and Zinn-Justin J eds., Les Houches Summer School Lectures LII, 1989 (North Holland, Amsterdam, 1991) p. 201.

[23] Berry M V and Tabor M 1976 Proc. Roy. Soc. Lond. 349 101; it ibid 1977 J. Phys. A: Math. Gen. 10

[24] Bogomolny E B in Quantum Dynamics of Simple Systems Oppo G L, Barnett S M, Riis E and Wilkinson M eds., The Forty Fourth Scottish Universities Summer School in Physics (Institute of physics publishing, Bristol and Philadelphia, August 1994) p 17.

[25] Keller J P 1962 J. Opt. Soc. Am. 52116

[26] Landau L D and Lifshitz E M 1958 Quantum mechanics, Course of theoretical Physics Vol. III (Pergamon Press)

[27] Berkolaiko G, Bogomolny E B and Keating J P 2001 J. Phys. A: Math. Gen. 34335

[28] Berkolaiko G and Keating J P 1999 J. Phys. A: Math. Gen. 327827 